\title{
Comparison of Two Types of Crossover Soave-Redlich-Kwong Equations of State for Derivative Properties of n-Alkanes
}

Vinhal, Andre Pinto Coelho Muniz; Jamali Rafsanjani, Asma; Behnejad, Hassan; Yan, Wei; Kontogeorgis, Georgios M.

Published in:

Industrial and Engineering Chemistry Research

Link to article, DOI:

10.1021/acs.iecr.9b05981

Publication date:

2020

Document Version

Peer reviewed version

Link back to DTU Orbit

Citation (APA):

Vinhal, A. P. C. M., Jamali Rafsanjani, A., Behnejad, H., Yan, W., \& Kontogeorgis, G. M. (2020). Comparison of Two Types of Crossover Soave-Redlich-Kwong Equations of State for Derivative Properties of n-Alkanes.

Industrial and Engineering Chemistry Research, 59(19), 9265-9278 . https://doi.org/10.1021/acs.iecr.9b05981

\section{General rights}

Copyright and moral rights for the publications made accessible in the public portal are retained by the authors and/or other copyright owners and it is a condition of accessing publications that users recognise and abide by the legal requirements associated with these rights.

- Users may download and print one copy of any publication from the public portal for the purpose of private study or research.

- You may not further distribute the material or use it for any profit-making activity or commercial gain

- You may freely distribute the URL identifying the publication in the public portal 


\section{Comparison of Two Types of Crossover Soave-Redlich-Kwong Equations of State for Derivative Properties of n-Alkanes}

Andre P. C. M. Vinhal, Asma Jamali Rafsanjani, Hassan Behnejad, Wei Yan, and Georgios M. Kontogeorgis

Ind. Eng. Chem. Res., Just Accepted Manuscript • DOI: 10.1021/acs.iecr.9b05981 • Publication Date (Web): 10 Apr 2020

Downloaded from pubs.acs.org on April 14, 2020

\section{Just Accepted}

"Just Accepted" manuscripts have been peer-reviewed and accepted for publication. They are posted online prior to technical editing, formatting for publication and author proofing. The American Chemical Society provides "Just Accepted" as a service to the research community to expedite the dissemination of scientific material as soon as possible after acceptance. "Just Accepted" manuscripts appear in full in PDF format accompanied by an HTML abstract. "Just Accepted" manuscripts have been fully peer reviewed, but should not be considered the official version of record. They are citable by the Digital Object Identifier (DOI®). "Just Accepted" is an optional service offered to authors. Therefore, the "Just Accepted" Web site may not include all articles that will be published in the journal. After a manuscript is technically edited and formatted, it will be removed from the "Just Accepted" Web site and published as an ASAP article. Note that technical editing may introduce minor changes to the manuscript text and/or graphics which could affect content, and all legal disclaimers and ethical guidelines that apply to the journal pertain. ACS cannot be held responsible for errors or consequences arising from the use of information contained in these "Just Accepted" manuscripts. 


\title{
Comparison of Two Types of Crossover Soave-
}

\section{Redlich-Kwong Equations of State for Derivative}

\author{
Properties of n-Alkanes
}

Andre P.C.M.Vinhal ${ }^{a}$, Asma Jamali ${ }^{a, b}$, Hassan Behnejad ${ }^{b}$, WeiYan $^{c}$, Georgios M. Kontogeorgis ${ }^{a^{*}}$

${ }^{a}$ Center for Energy Resources Engineering (CERE), Department of Chemical and Biochemical Engineering, Technical University of Denmark, DK-2800 Kgs. Lyngby, Denmark

${ }^{b}$ Department of Physical Chemistry, School of Chemistry, University College of Science, University of Tehran, Tehran 14155, Iran

${ }^{\mathrm{c} C e n t e r ~ f o r ~ E n e r g y ~ R e s o u r c e s ~ E n g i n e e r i n g ~(C E R E), ~ D e p a r t m e n t ~ o f ~ C h e m i s t r y, ~ T e c h n i c a l ~}$ University of Denmark, DK-2800 Kgs. Lyngby, Denmark

\footnotetext{
*Corresponding author

E-mail: gk@kt.dtu.dk, Tel.: +45 45252859
} 


\begin{abstract}
In this work, two different Crossover Soave-Redlich-Kwong ( $S R K)$ Equations of State $(E o S)$ were applied to incorporate the density fluctuations into the classical model for the representation of derivative properties of selected n-alkanes. Both methods, the first based on the renormalization of the Landau-Ginzburg expansion and the second based on the phase-space cell approximation, are capable of describing the non-classical behavior of the molar heat capacities and speed of sound close to the critical point. Nevertheless, the overall performance of the crossover equation is similar to the cubic model, since the non-mean-field $E o S$ reduce to the classical model far from the critical region. Additionally, the comparison of the crossover equations indicates that the Kernel term is useful for the correct representation of the heat capacity at constant volume, as well as other properties related to the weak divergence at the critical point, over a wider range of conditions.
\end{abstract}

\title{
INTRODUCTION
}

The accurate description of the thermodynamic properties of fluids is a major task in several industrial applications. In fact, the representation of relevant phase equilibria is fundamental for the design and control of operation of many processes in the oil and gas, chemical and other sectors [1]. Additionally, reliable theoretical methods are of great need in the industry, in order to substitute correlative models and to predict the experimental data over a wide range of compositions, temperatures and pressures [2]. Aside from predicting primary, i.e. phase equilibrium properties, it is also important to correctly describe secondary properties, like heat capacities and speed of sound. However, these properties are more difficult to predict [3], and traditional cubic EoS, like Peng-Robinson $(P R)$, which are frequently used in the oil and gas 
industry, fail to yield satisfactory results of the heat capacities in high pressure conditions, especially close to the critical point [4].

Classical EoS fail to describe the thermodynamic properties of fluids close to the critical point because they do not take into account the asymptotic and non-analytical behavior of fluids due to the critical fluctuations of density [5]. The non-classical behavior of fluids is characterized by universal scaling laws with universal exponents that are in accordance to the Renormalization Group $(R G)$ theory of the critical phenomena [6]. In order to represent the correct scaling behavior with EoS, it is necessary for a model to incorporate the fluctuations of the order parameter that arise in the critical region. Nonetheless, it is important that the model reduces to the classical analytic behavior far away from the critical point, where the density fluctuations are negligibly small [7]. There are two main $R G$ approaches in the literature [8] that take into account such inhomogeneities. The $R G$ corrections modify the Hamiltonian of a system into a renormalized one with reduced number of degrees of freedom, meaning that the microscopic degrees are integrated out as the characteristic length of the phenomenon becomes larger than the molecular interactions as the system approaches the critical point [5].

The first method considers an extension of the Landau-Ginzburg equation, where the fluctuations are characterized on a local mesoscopic Hamiltonian, in which the solution of the renormalization transformation to the classical equation are mapped to a cutoff point [9]. The application of this procedure is done by decomposing the expression for the free energy in an analytical part and a non-analytical part that contains the fluctuations. Then, the utilization of a truncated expansion in temperature and density variables is rescaled by crossover transformations that explicitly incorporates the known asymptotic critical scaling laws. Two versions of this approach are found in the literature. The first uses the truncated classical Landau expansion, as 
done by Chen et al. [9] and more recently by Wyczalkowska et al. [7] with the van der Waals EoS instead of the truncated Landau expansion, while the second uses a more phenomenological approach in which the critical non-analytical part is also represented by the classical $\operatorname{EoS}[10-16]$.

The second technique is based on Wilson's phase-space cell approximation which applies a block averaging procedure for the qualitative analysis of the critical phase behavior [6,17]. The method reduces the degrees of freedom by decomposing the system in a sequence of cells, or renormalized Hamiltonians, in which the fluctuations are averaged, allowing the calculation of correct values of the universal critical exponents. White and co-workers [18-22] used this idea to develop a global RG EoS that extends the range of application of the original model to model fluids both far away and close to the critical point [18-22], since the original method was restricted to the vicinity of the critical point [6].

Thus, the objective of this study is to provide a comprehensive overview of the capabilities of SRK EoS using two RG method to describe the singular behavior of derivative properties for $\mathrm{n}$ series alkanes near the critical point. Calculations have been performed over the defined temperature and pressure ranges and they are compared to the correlated experimental data [23]. In the process, the applicability of these models is considered by highlighting their strengths and weaknesses in representing the second order derivative properties.

We shall proceed as follows: In Section 2, we describe the crossover EoS using the two main methods to introduce density fluctuations in the classical thermodynamic model. In Section 3, we explain the parametrization procedure of the crossover $E o S$. The results are presented in Section 4, where we also compare the non-mean-field model with the classical $S R K E O S$, regarding the representation of the correlated experimental data. Finally, the last section contains the conclusions of this work. 


\section{CROSSOVER EQUATION OF STATE}

The classical thermodynamic model, to which density fluctuations are incorporated into, used in this work is $S R K E O S$ [24]. The pressure explicit expression of this model is given by:

$$
P=\frac{R T}{v-b}-\frac{a}{v(v+b)}
$$

where $P$ is the pressure, $v$ is the molar volume, and $R$ is the universal gas constant. The parameters $a$ and $b$ are repulsive and attractive term constants and are calculated with the following equations:

$$
\begin{aligned}
& a=\frac{0.4275 R^{2} T_{c}^{2}}{P_{c}}\left[1+k\left(1-\sqrt{\frac{T}{T_{c}}}\right)\right]^{2} \\
& b=\frac{0.08664 R T_{c}}{P_{c}}
\end{aligned}
$$

where $k$ is a function of the acentric factor $(\omega)$ defined as:

$$
k=0.480+1.574 \omega-0.175 \omega^{2}
$$

As mentioned previously, traditional $E o S$ fail to correctly describe the critical behavior of fluids because they do not take into account the density fluctuations that are significant close to the critical point. Therefore, in this work, we have applied two different crossover procedures based on the $R G$ theory, in order to bridge the gap between the non-classical critical behavior to classical behavior far from the critical point.

The first method is a phenomenological procedure proposed by Kiselev and co-workers, and it is based on the renormalization of a Landau expansion originally proposed by Chen et al. [9]. The second method is a recursive procedure developed by White and co-workers [19-22], which is an extension of the work of Wilson [6], as explained in the previous section. In the next subsections, we describe in detail the application of the two RG methods to obtain two different Crossover $S R K$ EoS. 


\section{Kiselev's method}

To apply Kiselev's method to an EoS, one must first rewrite the classical expression for the Helmholtz free energy in the dimensionless form, as given by:

$$
\bar{A}(T, v)=\Delta \bar{A}(\bar{\tau}, \bar{\eta})+\bar{A}^{r e s}\left(T, v_{0 c}\right)-\bar{\eta} \bar{P}_{0}(T)-\ln v_{0 c}+\bar{A}_{0}(T)-K(\tau, \eta)
$$

where $\bar{A}(T, v)=\frac{A(T, v)}{R T}$ is the total dimensionless Helmholtz energy, $\bar{P}_{0}(T)=P\left(T, v_{0 c}\right) v_{0 c} / R T$ is the dimensionless pressure at the critical isochore, $v_{0 c}$ is the classical critical volume, $\bar{A}_{0}(T)$ is the dimensionless temperature dependent part of Helmholtz free energy for an ideal gas, and $K(\tau, \eta)$ is the Kernel term, which is used to provide the correct representation of the isochoric heat capacity. The critical Helmholtz energy, $\Delta \bar{A}(\bar{\tau}, \bar{\eta})$, is given by:

$$
\Delta \bar{A}(\bar{\tau}, \bar{\eta})=\bar{A}^{\text {res }}(\bar{T}, \bar{v})-\bar{A}^{\text {res }}\left(\bar{T}, v_{0 c}\right)+\bar{\eta} \bar{P}_{0}(\bar{T})-\ln (\bar{\eta}+1)
$$

and $\bar{A}^{\text {res }}$ is the residual part of Helmholtz free energy which is a function of the renormalized temperature and volume, i.e. $\bar{T}=T_{0 c}(1+\bar{\tau})$ and $\bar{v}=v_{0 c}(1+\bar{\eta})$, and are calculated by the expressions:

$$
\begin{aligned}
& \bar{\tau}=\tau Y^{\left(-\frac{\alpha}{2 \Delta}\right)}+(1+\tau) \Delta \tau_{c} Y^{\left(\frac{2(2-\alpha)}{3 \Delta}\right)} \\
& \bar{\eta}=\eta Y^{\left(\frac{\gamma-2 \beta}{4 \Delta}\right)}+(1+\eta) \Delta \eta_{c} Y^{\left(\frac{2-\alpha}{2 \Delta}\right)}
\end{aligned}
$$

where, $\gamma=1.24, \beta=0.325, \alpha=2-\gamma-2 \beta=0.110$ and $\Delta=0.51$ are the universal nonclassical critical exponents and $T_{0 c}$ is the classical critical temperature. The terms $\Delta \tau_{c}=$ $\left(T_{c} / T_{0 c}-1\right)$ and $\Delta \eta_{c}=\left(v_{c} / v_{0 c}-1\right)$ are the dimensionless shifts of the critical parameters, while $\tau=T / T_{c}-1$ and $\eta=v / v_{c}-1$ are the dimensionless distance from the real critical temperature and volume $\left(T_{c}\right.$ and $\left.v_{c}\right)$, respectively. 
The function $Y(q)$ plays an important role in the crossover $E o S$, and it is related to the parametric variable $q$, which represents the distance from the critical point. These two terms are calculated as the following:

$$
\begin{aligned}
& Y(q)=\left(\frac{q}{1+q}\right)^{2 \Delta} \\
& \left(q^{2}-\frac{\tau}{G i}\right)\left[1-\frac{p^{2}}{4 b^{2}}\left(1-\frac{\tau}{q^{2} G i}\right)\right]=\left\{\frac{b\left(\eta\left(1+v_{1} e^{-10 \eta}\right)+d_{1} \tau\right)}{m_{0} G i^{\beta}}\right\}^{2} Y^{(1-2 \beta) / \Delta}
\end{aligned}
$$

where $G i$ is the Ginzburg number, $v_{1}$ and $d_{1}$ are the system dependent parameters, $p^{2}$ and $b^{2}$ can be set equal the universal linear model parameter $p^{2}=b^{2} \cong 1.359$, and to reduce the number of adjustable parameters, $m_{0}$ is considered as a universal parameter equal to one.

Finally, the Kernel term is defined as:

$$
K(\tau, \eta)=\frac{1}{2} a_{20} \tau^{2}\left[Y^{-\frac{\alpha}{\Delta}}-1\right]+\frac{1}{2} a_{21} \tau^{2}\left[Y^{-\frac{(\alpha-\Delta)}{\Delta}}-1\right]
$$

where the coefficients $a_{20}$ and $a_{21}$ are calculated along the critical isochore at $\tau>0$. The Kernel term is responsible for the divergent behavior of the isochoric heat capacity [25].

The crossover SRK EoS using Kiselev's method is obtained by applying Eq. (1) to obtain Eq. (6), then the pressure explicit equation is obtained by differentiation of Eq. (6) with respect to volume, yielding:

$$
P=-\left(\frac{\partial A}{\partial v}\right)_{T}=-\frac{R \bar{T}}{v_{c}}\left(\frac{\partial \Delta \bar{A}(\bar{\tau}, \bar{\eta})}{\partial \eta}\right)_{T}+\bar{P}_{0}(T)-v_{0 c}^{-1}\left(\frac{\partial K}{\partial \eta}\right)_{T}
$$

The details of this crossover procedure can be found in the references $[13,14]$.

\section{White's method}

One of the first authors to apply White's recursive procedure to a cubic $E o S$ were Cai and Prausnitz [26], who have used the $S R K E O S$ as the underlying model to which the density fluctuations are 
introduced. The recursive procedure for a pure fluid concerns of correcting the classical free energy density in the following way:

$$
\begin{aligned}
& f=f_{n}-\alpha \rho^{2} \\
& f_{n}=f_{n-1}+\delta f_{n} \\
& \delta f_{n}=-K_{n} \ln \left(\frac{\Omega_{n}^{s}}{\Omega_{n}^{l}}\right) \\
& K_{n}=\frac{k_{b} T}{2^{3 n} L^{3}} \\
& \Omega_{n}^{\lambda}=\int_{0}^{\min \left(\rho, \rho_{\max }-\rho\right)} \exp \left[-\frac{G_{n}^{\lambda}(\rho, y)}{K_{n}}\right] d y \\
& G_{n}^{\lambda}=0.5\left[\overline{f_{n}^{\lambda}}(\rho+y)+\overline{f_{n}^{\lambda}}(\rho-y)-2 \overline{f_{n}^{\lambda}}(\rho)\right] \\
& \overline{f_{n}^{l}}=f_{n-1}+\alpha \rho^{2} \\
& \overline{f_{n}^{s}}=f_{n-1}+\alpha \rho^{2} \frac{\phi}{2^{2 n}}
\end{aligned}
$$

where the subscript $n$ and the superscript $\lambda$ represent the step in the recursive procedure and the long-range ( $l$ ) and short-range $(s)$ correlations, respectively; $k_{b}$ is the Boltzmann constant, $T$ is the temperature, $\alpha$ is a term related to the attractive Helmholtz energy density, $\rho$ is the density, $\rho_{\max }$ is the maximum packing density in this work approximated to $0.999 b^{-1}, L$ and $\phi$ are parameters of the recursive procedure and represent the cut-off length and a function of the initial shortest wavelength of density fluctuations.

White's recursive procedure starts with the free energy density containing only the repulsive, i.e. short-range interactions, between the molecules, meaning that in the first step $f_{0}=f_{\text {rep }}$. However, according to Cai and Prausnitz [26], the separation of the attractive and repulsive interactions in a phenomenological model like the $S R K E O S$ is not clear. In other words, the first term on the right-hand side of Eq. (1) is not equivalent to the hard-sphere model [27]. Therefore, 
the authors used experimental data to fit $R G$ calculations, concluding that the attractive part of the free energy $\left(\alpha \rho^{2}\right)$ corresponds to approximately:

$$
-\alpha \rho^{2}=-\frac{1}{2} a \rho^{2}
$$

where $a$ is the attractive term constant of the SRK model calculated by Eq. (2). Consequently, the repulsive free energy density is given by $f_{S R K}$, i.e. the free energy density estimated with Eq. (1), subtracting the attractive contributions, which yields:

$$
f_{0}=f_{S R K}+\frac{1}{2} a \rho^{2}
$$

One of the limitations of the procedure adopted by Cai and Prausnitz [26], in order to accommodate the phenomenological character of the $S R K E O S$, is that the short-range correlation is not achieving its final form as $n$ goes to infinity. It means that not all shortwave lengths are incorporated into the crossover equation, which limits the capacity of the model to represent properties like the heat capacity at constant volume and other weakly divergent thermodynamic properties, which mean-field models are unable to correctly describe [25]. This can be seen by substituting Eqs. (21) and (22) in the recursive procedure given by Eqs. (13) to (20). In the first step the corrected free energy densities are equal to:

$$
\begin{aligned}
& \overline{f_{1}^{l}}=f_{S R K}+a \rho^{2} \\
& \overline{f_{1}^{s}}=f_{S R K}+\frac{1}{2} a \rho^{2}\left(1+\frac{\phi}{2^{2}}\right)
\end{aligned}
$$

After $n$ iterations the values of the previous functions are:

$$
\begin{aligned}
& \overline{f_{n}^{l}}=f_{S R K}+a \rho^{2}+\sum \delta f_{n} \\
& \overline{f_{n}^{s}}=f_{S R K}+\frac{1}{2} a \rho^{2}\left(1+\frac{\phi}{2^{2 n}}\right)+\sum \delta f_{n}
\end{aligned}
$$

where we have used the expression $f_{n}=f_{0}+\sum \delta f_{n}$ [21] to obtain the corrected short and longrange densities, i.e. Eqs. (25) and (26), at the final summand of the recursive procedure the $n^{\text {th }}$ 
order summation. This expression is defined by a simple manipulation of the recursive procedure given by Eq. (12) and it indicates that $n^{\text {th }}$ order approximation to the free energy density is the sum of the initial free energy density and a sequence of the fluctuation contributions up to the order $n$.

The analysis of Eq. (24) shows that as $n \rightarrow \infty$, the term $\frac{\phi}{2^{2 n}}$ becomes negligible; yet, an attraction contribution is still present in the short-range part of the free energy density. This limits the incorporation of increasingly longer wavelength density fluctuations as the order is increased, because an attractive term is added to $\Omega_{n}^{s}$ and $\frac{\Omega_{\mathrm{n}}^{\mathrm{s}}}{\Omega_{\mathrm{n}}^{1}}$ in Eq. (15), which represents the ratio of nonmean-field contributions to mean field contributions, does not yield the full free energy density correction $\left(\delta f_{n}\right)$, obtained from the recursion relations in the infinite order limit.

In order to correct this limitation, we defined $f_{0}=f_{S R K}$ and the attractive part of the recursive procedure, i.e. $\alpha \rho^{2}$, equal to $a \rho^{2}$. With these modifications, the corrected free energy densities, in the first step of the recursive procedure, are equal to:

$$
\begin{aligned}
& \overline{f_{1}^{l}}=f_{S R K}+a \rho^{2} \\
& \overline{f_{1}^{s}}=f_{S R K}+a \rho^{2} \frac{\phi}{2^{2}}
\end{aligned}
$$

After $n$ summands the values of the previous functions are:

$$
\begin{aligned}
& \overline{f_{n}^{l}}=f_{S R K}+a \rho^{2}+\sum \delta f_{n} \\
& \overline{f_{n}^{s}}=f_{S R K}+a \rho^{2} \frac{\phi}{2^{2 n}}+\sum \delta f_{n}
\end{aligned}
$$

The rest of the procedure is similar to the one developed by Cai and Prausnitz [26], and is given by Eqs. (13) to (18).

The comparison of Eqs. (23) and (24) with (27) and (28) indicates the modified procedure is similar for the long-range correction term of the free energy, but differs for the short-range 
contributions, allowing the complete introduction of the density fluctuations and improving the representation of the properties that have a weak divergence at the critical point. In fact, this procedure is the same as the one used in SAFT implementations of White's recursive procedure $[8,28]$ and it is similar to White's original development $[18-20,22]$. The difference is related to the interpretation of the critical phenomena.

In White's original method, $L$ is defined as the cutoff length separating the repulsive and attractive contributions of the free energy, and $-a \rho^{2}$ is an approximation of the attractive interaction. In the usual SAFT implementation, $L$ separates the mean-field and non-mean-field part of the free energy density, hence it is assumed that the initial $f_{0}$, calculated with a $E o S$ accurately describes the behavior of a system far away from the critical point. Apart from the differences in the interpretation, both approaches yield similar equations for the corrected free energy densities as described by Forte et al [28,29].

The application of the crossover $S R K$ using White's procedure to represent the thermodynamic properties of fluids requires the numerical solution of the total free energy of the system. This is done by dividing the density range of the fluid in a pre-defined number of intervals. Following other works [21-23], we used 500 density points, from $\rho=\frac{10^{-12}}{b}$ to $\rho=\frac{1}{0.999 b}$. Besides, a spline function is used to estimate $f(\rho)$ and five iterations were used in the numerical procedure for both procedures of White's method for most of the calculations performed in this work.

\section{PARAMETRIZATION OF THE CROSSOVER EOS}

In a previous work [30], we have thoroughly described the parametrization of the crossover $E o S$ mentioned previously for the representation of the phase equilibria of pure n-alkanes (from methane to n-decane). In the case of White's procedure, we have kept the classical parameters that 
were tuned to match the pure components' vapor pressure and liquid and vapor density curves both far away from and close to the critical point, i.e. the classical critical pressure $\left(P_{0 c}\right)$, temperature ( $\left.T_{0 c}\right)$ and acentric factor $\left(\omega_{0}\right)$. Nonetheless we have changed the value of the cutoff length $(L)$, due to the modification of the recursive procedure as mentioned in the previous section. Although no significant changes are seen far from the critical point since the density fluctuations are negligible, the inclusion of additional short-range fluctuations requires refitting $L$ for a correct description of the critical region. In fact, the values for the cutoff length are larger than the ones obtained previously, but follow a similar trend (Table 1 and Fig. 1). The crossover SRK EoS using the recursive procedure similar to Cai and Praunistz [26] is referred to as CSRK while the model using the modified procedure developed in this work is referred to as CSRK2.

Table 1. The values of the crossover parameters for the crossover SRK EoS using White's modified procedure (CSRK2) for the selected n-alkanes.

\begin{tabular}{|lccccl|}
\hline Comp. & $T_{0 c}(\mathrm{~K})$ & $P_{0 c}($ bar $)$ & $\omega_{0}(-)$ & $L(\AA)$ & $\phi(-)$ \\
\hline $\mathrm{C}_{1}$ & 197.7 & 49.88 & -0.049 & 6.454 & 2.000 \\
\hline $\mathrm{C}_{2}$ & 317.1 & 54.75 & 0.033 & 6.932 & 2.000 \\
\hline $\mathrm{C}_{3}$ & 383.3 & 47.65 & 0.086 & 7.575 & 2.000 \\
\hline $\mathrm{C}_{4}$ & 441.4 & 43.00 & 0.128 & 8.139 & 2.000 \\
\hline $\mathrm{C}_{5}$ & 488.0 & 38.63 & 0.174 & 8.431 & 2.000 \\
\hline $\mathrm{C}_{6}$ & 527.1 & 35.08 & 0.221 & 8.775 & 2.000 \\
\hline $\mathrm{C}_{7}$ & 560.5 & 32.10 & 0.271 & 9.052 & 2.000 \\
\hline $\mathrm{C}_{8}$ & 591.0 & 29.83 & 0.315 & 9.174 & 2.000 \\
\hline $\mathrm{C}_{9}$ & 618.4 & 27.58 & 0.354 & 9.504 & 2.000 \\
\hline $\mathrm{C}_{10}$ & 639.4 & 25.50 & 0.411 & 9.876 & 2.000 \\
\hline
\end{tabular}




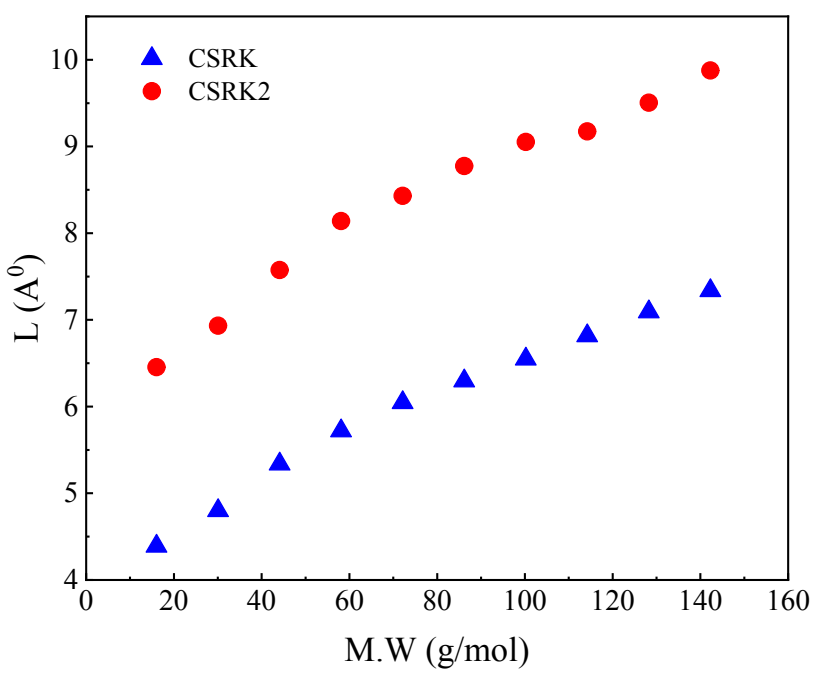

Fig. 1. Cut-off length for the crossover $S R K E O S$ using White's procedure as a function of the molecular weight for selected n-alkanes. The blue triangles represents the optimum values with the approach given in [30] and the red circles are the ones obtained in this work.

On the other hand, the vapor pressure and coexistence diagrams are similar showing that for phase equilibrium calculations, the modified procedure does not substantially modify the representation of the experimental data (Fig. 2). Nevertheless, the same is not valid for the derivative properties, which will be addressed in the next section. 


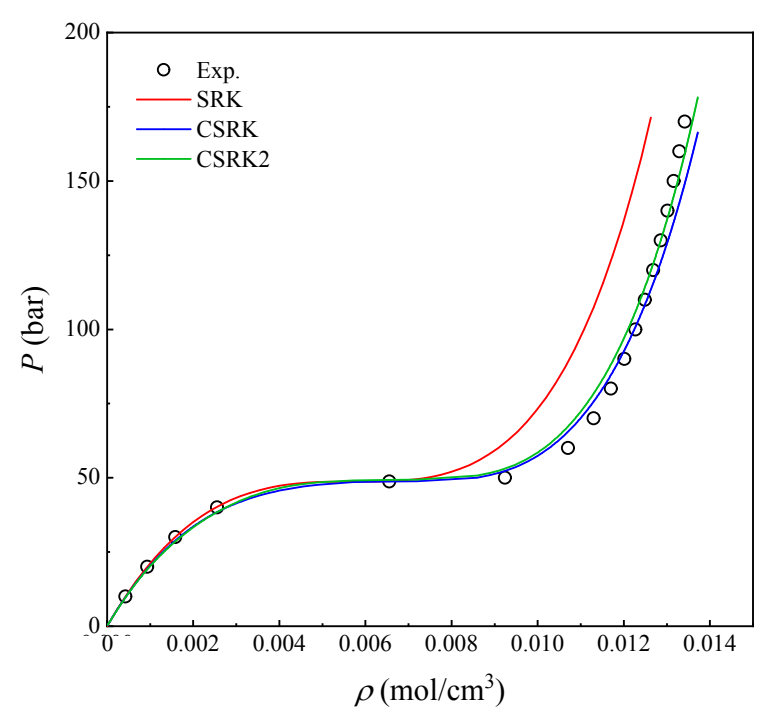

(a)

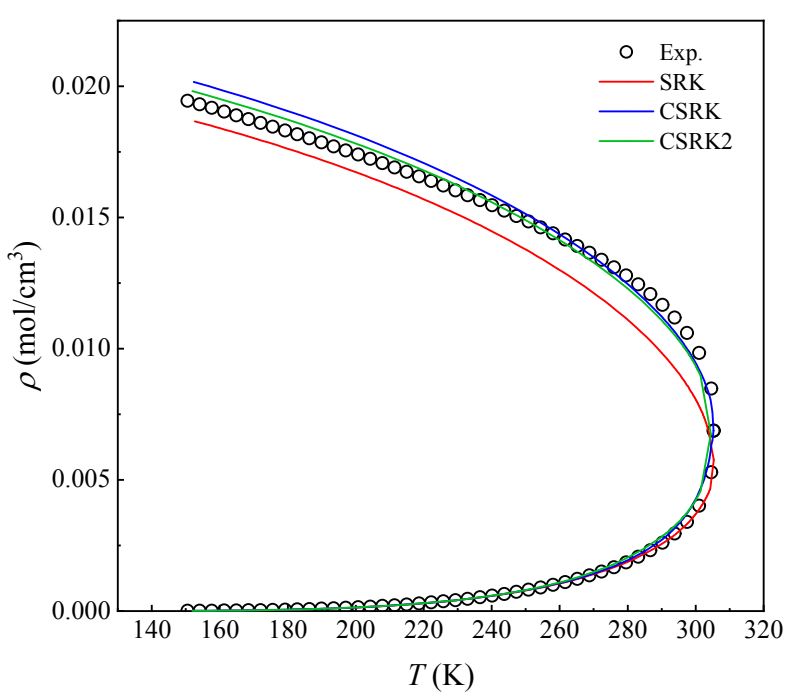

(b)

Fig. 2. Critical isotherm (a) and coexistence diagram (b) of ethane calculated with $S R K$ and two versions of the crossover $S R K$ using White's approach.

In the case of Kiselev's method, the procedure is similar to the one described in the previous work; however, we have added the Kernel term to improve the results for the derivative properties. This is an additive renormalization term given in Eq. (6), responsible for the divergent behavior of the isochoric heat capacity [25,31]. As seen in Eq. (11), it has two additional parameters, $a_{20}$ and $a_{21}$, which are defined by fitting the critical isochore of the pure fluid. Table 2 shows the values of these parameters for the n-alkanes studied in this work. After the parametrization procedure, we have applied the models to describe some derivative properties and compared the crossover models with the classical $S R K E o S$, as well as experimental data. 
experimental data for the saturated liquid phase, while it underpredicts the points for the saturated vapor phase and the supercritical fluid.

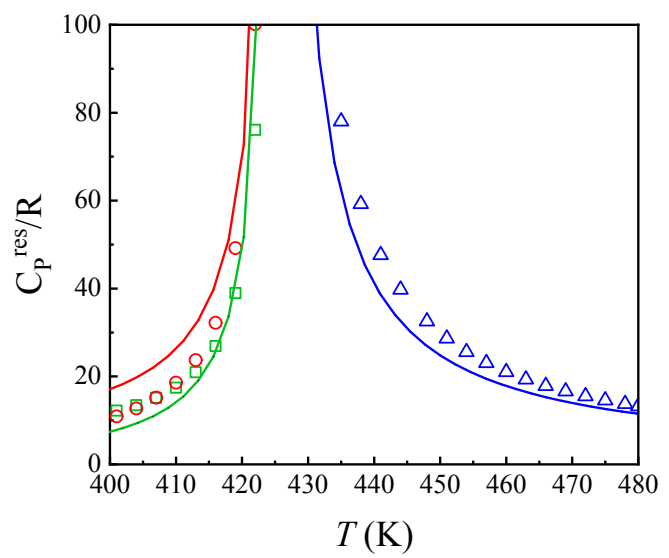

Fig. 3. The correlated experimental $C_{p}{ }^{r e s} / R$ for n-butane taken from [23], and the calculated curves using $S R K E O S$. The red and green lines and points represent the saturated vapor and liquid phases, respectively, while the blue lines and points describe the supercritical fluid at the critical volume.

In an attempt to evaluate the improvements obtained with the introduction of the density fluctuations in the classical model, we have applied the crossover $S R K E O S$, using both White and Kiselev's approach to describe $C_{p}{ }^{r e s} / R$ of n-butane. Fig. 4 shows the results obtained using two approaches of the recursive method to incorporate density fluctuations in the classical model. The comparison of Figs. 3 and 4 indicates that $R G$ corrections of the $S R K E O S$ do not substantially improve the representation of experimental $C_{p}$. Additionally, both approaches of the recursive method yield similar results. Finally, the crossover $S R K$ EoS using Kiselev's method was applied for the representation of the same data (Fig. 5). This non-mean-field model also yields to analogous estimations for $C_{p}{ }^{r e s} / R$; however, this method has less accuracy than the classical SRK EoS and both approaches of recursive method by approaching to the critical region. 


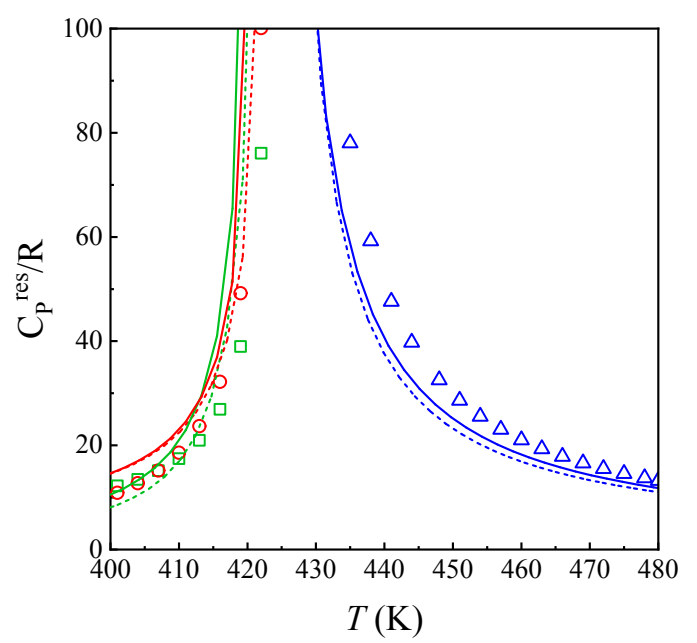

Fig. 4. The correlated experimental $C_{p}{ }^{r e s} / R$ for n-butane taken from [23], as well as the calculated curves using the crossover SRK EOS with the two approaches of the White's procedure. The dashed and solid lines correspond to CSRK and CSRK2, respectively. The red and green lines and points represent the saturated vapor and liquid phases, respectively, while the blue lines and points describe the supercritical fluid at the critical volume.

In addition to calculations of $C_{p}{ }^{r e s} / R$ at the critical volume, we have applied the aforementioned models to calculate the same property at different pressures close to the critical point. Fig. 6 contains the calculated curves using the mean-field SRKEOS and the non-mean-field model with Kiselev's method. It is possible to observe from the figure that both models yield alike results and are capable of describing this property close to the critical point. The same behavior is seen with the application of White's method (Fig. 7); nonetheless, as the critical point is slightly modified, a shift of the curves is observed, especially for the heavier hydrocarbon. In fact, this difference is related to the parametrization procedure, which, as discussed in a previous paper [30], is done by fitting of the saturated pressure, liquid and vapor phase volume curves, and PVT data at the critical isotherm. Finally, Table 3 shows the average absolute deviations $(A A D \%)$ of the molar heat 
capacity $\left(C_{p}\right)$. In this case, the low $A A D \%$ of the classical model (Table 3 ) is a direct consequence of the ideal $C_{p}$ correlation, which corresponds to a large amount of the $C_{p}$ of a substance [30].

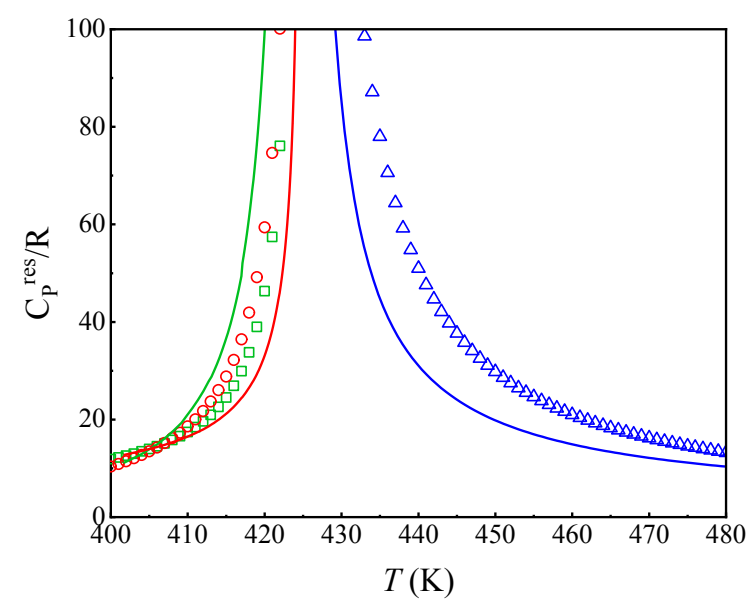

Fig. 5. The correlated experimental $C_{p}^{r e s} / R$ for n-butane taken from [23], as well as the calculated curves using Kiselev's method to correct $S R K$. The red and green lines and points represent the saturated vapor and liquid phases, respectively, while the blue lines and points describe the supercritical fluid at the critical volume.

Table 3. Calculated deviations for the molar heat capacity at constant pressure $\left(C_{P}\right)$ for different pressures using different crossover methods applied on $S R K E O S$ in comparison to classical $S R K$ EoS. 


\begin{tabular}{|c|c|c|c|c|c|c|}
\hline Compound & $\begin{array}{l}\mathrm{P} \\
(\mathrm{MPa})\end{array}$ & $\begin{array}{l}\text { AAD\% } \\
\text { SRK }\end{array}$ & $\begin{array}{l}\text { AAD\% } \\
\text { Kiselev's } \\
\text { method }\end{array}$ & $\begin{array}{l}\text { AAD\% } \\
\text { White's } \\
\text { method } \\
\text { CSRK }\end{array}$ & $\begin{array}{l}\text { AAD\% } \\
\text { White's } \\
\text { method } \\
\text { CSRK2 } \\
\end{array}$ & $\begin{array}{l}\text { T-range } \\
(\mathrm{K})\end{array}$ \\
\hline \multirow{4}{*}{$\mathrm{C} 1$} & 4.5992 & 8.00 & 2.8 & 4.1 & 3.4 & \multirow{4}{*}{$170-240$} \\
\hline & 4.8 & 8.2 & 3.6 & 11.4 & 4.6 & \\
\hline & 5.1 & 8.0 & 4.4 & 3.6 & 3.8 & \\
\hline & 5.4 & 8.0 & 5.4 & 3.9 & 3.9 & \\
\hline \multirow{4}{*}{$\mathrm{C} 2$} & 4.8718 & 10.4 & 14.5 & 4.0 & 6.8 & \multirow{4}{*}{$280-350$} \\
\hline & 5.0 & 10.9 & 6.2 & 5.8 & 7.7 & \\
\hline & 5.3 & 10.6 & 4.8 & 6.5 & 8.4 & \\
\hline & 5.6 & 10.7 & 5.6 & 7.2 & 8.1 & \\
\hline \multirow{4}{*}{ C3 } & 4.2476 & 6.8 & 4.3 & 3.4 & 10.8 & \multirow{4}{*}{$350-440$} \\
\hline & 4.5 & 6.9 & 2.9 & 11.0 & 15.0 & \\
\hline & 4.8 & 7.2 & 3.4 & 5.4 & 13.1 & \\
\hline & 5.1 & 7.3 & 3.9 & 6.2 & 11.4 & \\
\hline \multirow{4}{*}{$\mathrm{C} 4$} & 3.7960 & 8.1 & 2.1 & 10.0 & 13.4 & \multirow{4}{*}{$400-480$} \\
\hline & 4.0 & 8.2 & 3.4 & 10.4 & 22.6 & \\
\hline & 4.3 & 8.3 & 4.8 & 9.3 & 17.4 & \\
\hline & 4.6 & 3.8 & 5.3 & 9.0 & 14.5 & \\
\hline \multirow{4}{*}{ C5 } & 3.37 & 8.3 & 4.2 & 9.3 & 16.1 & \multirow{4}{*}{$450-520$} \\
\hline & 4.0 & 8.9 & 6.4 & 10.1 & 19.3 & \\
\hline & 4.3 & 8.9 & 7.5 & 10.0 & 16.2 & \\
\hline & 4.6 & 8.8 & 8.3 & 9.7 & 13.8 & \\
\hline \multirow{4}{*}{ C6 } & 3.034 & 9.3 & 7.2 & 16.3 & 21.4 & \multirow{4}{*}{$480-540$} \\
\hline & 3.3 & 9.5 & 6.0 & 16.4 & 32.4 & \\
\hline & 3.6 & 9.5 & 7.5 & 14.6 & 25.1 & \\
\hline & 3.9 & 9.2 & 8.8 & 12.9 & 19.4 & \\
\hline \multirow{4}{*}{ C7 } & 2.7360 & 8.5 & 2.6 & 13.4 & 27.6 & \multirow{4}{*}{$530-580$} \\
\hline & 3.0 & 9.0 & 4.9 & 16.6 & 35.0 & \\
\hline & 3.3 & 9.0 & 6.0 & 14.8 & 27.6 & \\
\hline & 3.6 & 8.6 & 7.0 & 13.4 & 21.5 & \\
\hline \multirow{4}{*}{ C8 } & 2.497 & 9.5 & 3.3 & 13.6 & 25.0 & \multirow{4}{*}{$550-600$} \\
\hline & 2.7 & 9.6 & 4.7 & 18.2 & 38.4 & \\
\hline & 3.0 & 9.4 & 5.2 & 15.0 & 29.1 & \\
\hline & 3.3 & 8.4 & 5.9 & 12.2 & 20.7 & \\
\hline \multirow{4}{*}{ C10 } & 2.103 & 11.4 & 4.5 & 15.4 & 21.6 & \multirow{4}{*}{$590-650$} \\
\hline & 2.4 & 11.3 & 7.6 & 19.6 & 32.9 & \\
\hline & 2.7 & 10.7 & 8.9 & 33.3 & 24.2 & \\
\hline & 3.0 & 8.6 & 7.8 & 21.8 & 16.3 & \\
\hline Average & & 8.8 & 5.6 & 11.6 & 18.0 & \\
\hline
\end{tabular}


Table 4. Calculated deviations for the molar heat capacity at constant volume $\left(C_{v}\right)$ for different pressures using different crossover methods applied on $S R K E O S$ in comparison to classical SRK EoS.

\begin{tabular}{|c|c|c|c|c|c|c|}
\hline Compound & P (MPa) & $\begin{array}{l}\text { AAD \% } \\
\text { SRK }\end{array}$ & $\begin{array}{l}\text { AAD\% } \\
\text { Kiselev's } \\
\text { method }\end{array}$ & $\begin{array}{l}\text { AAD\% } \\
\text { White's } \\
\text { method } \\
\text { CSRK }\end{array}$ & $\begin{array}{l}\text { AAD\% } \\
\text { White's } \\
\text { method } \\
\text { CSRK2 }\end{array}$ & $\begin{array}{l}\text { T-range } \\
(\mathrm{K})\end{array}$ \\
\hline \multirow{4}{*}{$\mathrm{C} 1$} & 4.5992 & 6.9 & 4.7 & 7.1 & 5.6 & \multirow{4}{*}{$170-240$} \\
\hline & 4.8 & 7.5 & 5.7 & 7.7 & 6.1 & \\
\hline & 5.1 & 7.6 & 7.1 & 7.8 & 6.1 & \\
\hline & 5.4 & 7.5 & 7.9 & 7.8 & 6.0 & \\
\hline \multirow{4}{*}{$\mathrm{C} 2$} & 4.8718 & 6.8 & 4.2 & 6.7 & 5.1 & \multirow{4}{*}{$280-350$} \\
\hline & 5.0 & 7.0 & 4.1 & 7.1 & 5.3 & \\
\hline & 5.3 & 7.3 & 4.8 & 7.2 & 5.4 & \\
\hline & 5.6 & 7.3 & 5.7 & 7.2 & 5.5 & \\
\hline \multirow{4}{*}{$\mathrm{C} 3$} & 4.2476 & 4.3 & 2.0 & 4.7 & 3.7 & \multirow{4}{*}{$350-440$} \\
\hline & 4.5 & 4.6 & 2.0 & 5.1 & 3.9 & \\
\hline & 4.8 & 4.6 & 2.1 & 5.0 & 3.9 & \\
\hline & 5.1 & 4.4 & 2.3 & 4.9 & 3.8 & \\
\hline \multirow{4}{*}{$\mathrm{C} 4$} & 3.7960 & 3.4 & 1.3 & 3.6 & 2.9 & \multirow{4}{*}{$400-480$} \\
\hline & 4.0 & 3.6 & 1.9 & 3.8 & 2.9 & \\
\hline & 4.3 & 3.6 & 2.5 & 3.7 & 2.8 & \\
\hline & 4.6 & 1.4 & 1.0 & 3.6 & 2.7 & \\
\hline \multirow{4}{*}{ C5 } & 3.37 & 2.9 & 1.5 & 3.1 & 2.4 & \multirow{4}{*}{$450-520$} \\
\hline & 4.0 & 3.1 & 2.7 & 3.3 & 2.4 & \\
\hline & 4.3 & 3.0 & 2.8 & 3.2 & 2.4 & \\
\hline & 4.6 & 2.8 & 2.7 & 2.9 & 2.2 & \\
\hline \multirow{4}{*}{ C6 } & 3.034 & 2.8 & 1.5 & 2.8 & 2.2 & \multirow{4}{*}{$480-540$} \\
\hline & 3.3 & 2.8 & 2.2 & 2.8 & 2.2 & \\
\hline & 3.6 & 2.7 & 2.5 & 2.7 & 1.9 & \\
\hline & 3.9 & 2.4 & 2.4 & 2.4 & 1.8 & \\
\hline \multirow{4}{*}{ C7 } & 2.7360 & 2.6 & 0.7 & 3.3 & 2.6 & \multirow{4}{*}{$530-580$} \\
\hline & 3.0 & 2.5 & 1.4 & 3.3 & 2.4 & \\
\hline & 3.3 & 2.3 & 1.7 & 3.0 & 2.0 & \\
\hline & 3.6 & 2.1 & 1.7 & 2.6 & 1.7 & \\
\hline \multirow{4}{*}{$\mathrm{C} 8$} & 2.497 & 2.5 & 1.7 & 2.9 & 2.1 & \multirow{4}{*}{$550-600$} \\
\hline & 2.7 & 2.4 & 1.8 & 2.9 & 2.0 & \\
\hline & 3.0 & 2.1 & 1.8 & 2.5 & 1.6 & \\
\hline & 3.3 & 1.7 & 1.8 & 2.0 & 1.3 & \\
\hline \multirow{4}{*}{$\mathrm{C} 10$} & 2.103 & 2.4 & 0.9 & 2.7 & 2.3 & \multirow{4}{*}{$590-650$} \\
\hline & 2.4 & 2.3 & 1.5 & 2.6 & 1.9 & \\
\hline & 2.7 & 1.9 & 1.5 & 2.5 & 1.5 & \\
\hline & 3.0 & 1.4 & 1.4 & 1.8 & 1.1 & \\
\hline Average & & 3.8 & 2.7 & 4.1 & 3.1 & \\
\hline
\end{tabular}




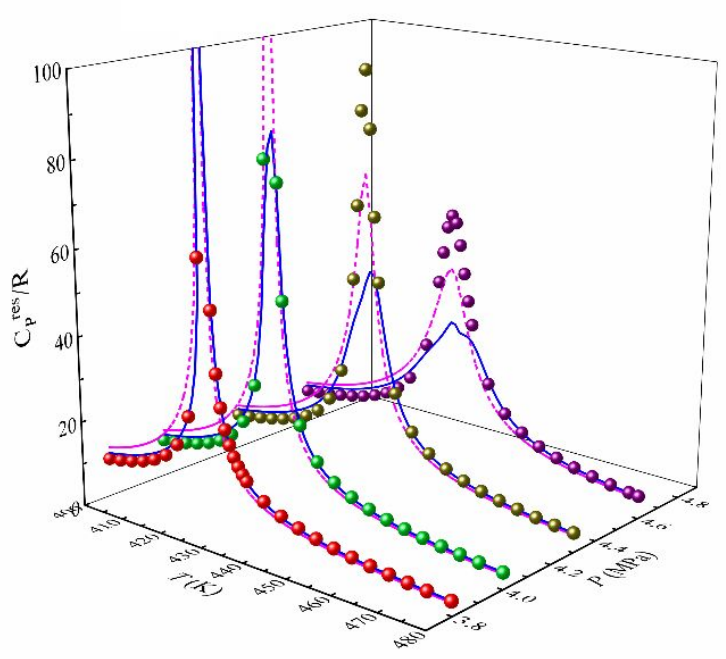

Fig. 6. The correlated experimental $C_{p}{ }^{\text {res }} / R$ for n-butane at the pressures shown in Table 3 taken from [23]. The dashed lines correspond to the classical $S R K E O S$ and the solid lines to the crossover model with Kiselev's method.

Following the representation of $C_{P}$, we have applied the models to represent $C_{v}$. The latter property has a weak divergence at the critical point, which classical models are usually unable to correctly represent. In fact, the heat capacity at constant volume does not diverge in the mean-field approximation. Unlike $C_{P}$, which as a function of the isothermal compressibility, shows strong divergence caused by the long-range interactions between the particles of the system, the internal energy and its derivatives are determined by the short-range behavior of the short-range nature of the intermolecular potential [25]. Fig. 8 shows the dimensionless residual heat capacity at constant volume $\left(C_{v}^{r e s} / R\right)$ for $\mathrm{n}$-butane. The plot indicates that the model is unable to represent the divergence of the property close to the critical point. 


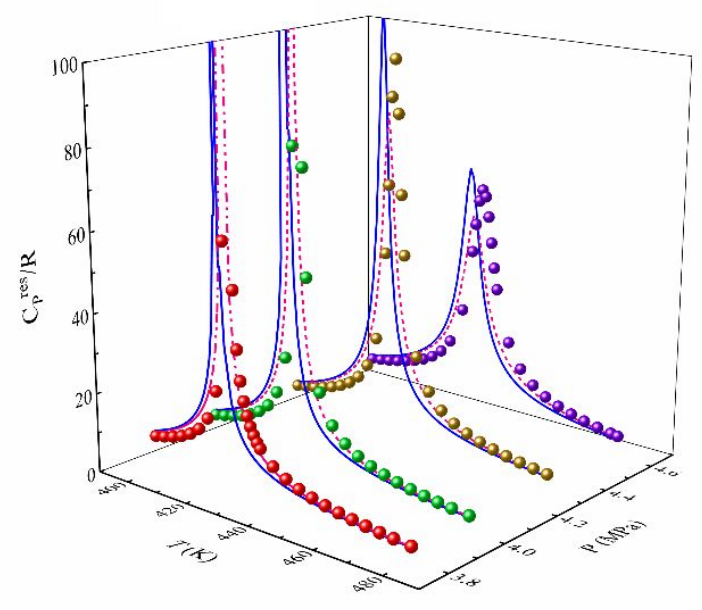

Fig. 7. The correlated experimental $C_{p}{ }^{r e s} / R$ for n-butane at the pressures shown in Table 3 taken from [23]. The dashed and solid lines correspond to CSRK and CSRK2, respectively.

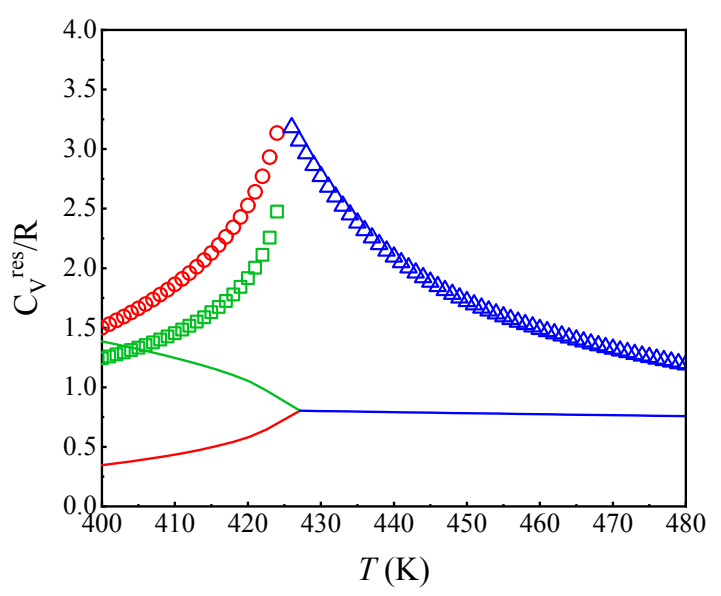

Fig. 8. The correlated experimental $C_{v}{ }^{r e s} / R$ for n-butane taken from [23], as well as the calculated curves using $S R K$. The red and green lines and points represent the saturated vapor and liquid phases, respectively, while the blue lines and points describe the supercritical fluid at the critical volume. 
The same behavior is observed for CSRK (Fig. 9). Due to the addition of an attractive term on the short-range correction of the free energy density in the recursive procedure (Eqs. 22 and 24), which limits the incorporation of short wavelengths in the classical model, the representation of $C_{v}^{r e s}$ is similar to the classical model. However, this limitation is corrected with the modified recursive procedure, yielding a superior representation of this property (Fig. 10).

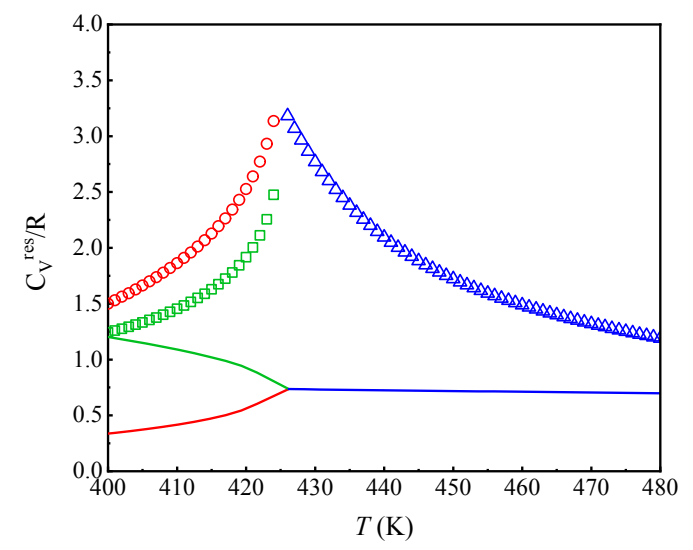

Fig. 9. The correlated experimental $C_{v}{ }^{r e s} / R$ for n-butane taken from [23], as well as the calculated curves using CSRK. The red and green lines and points represent the saturated vapor and liquid phases, respectively, while the blue lines and points describe the supercritical fluid at the critical volume.

The calculations of the dimensionless residual $C_{v}$ were also done with the crossover SRKEOS using Kiselev's method. Fig. 11 shows the experimental values and the calculated curves, indicating that this equation is also capable of representing the weak divergence of this property. 


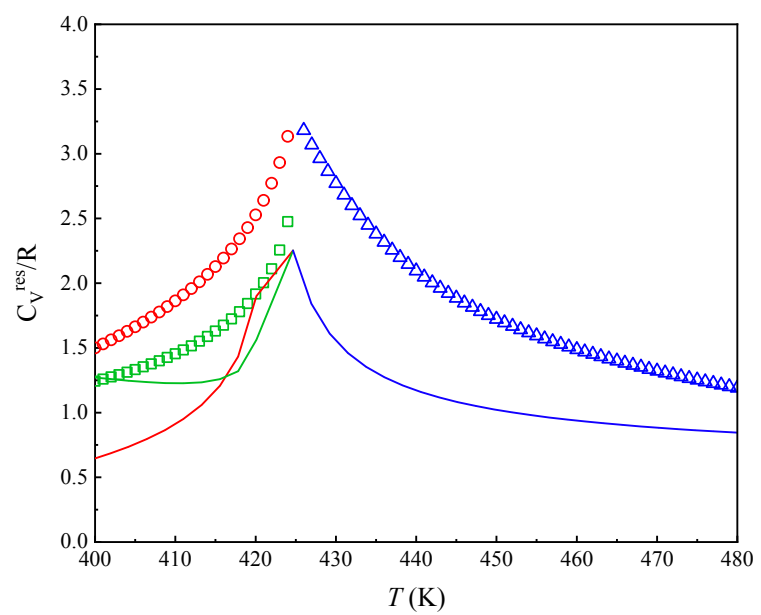

Fig. 10. The correlated experimental $C_{v}{ }^{r e s} / R$ for n-butane taken from [23], as well as the calculated curves using $C S R K 2$. The red and green lines and points represent the saturated vapor and liquid phases, respectively, while the blue lines and points describe the supercritical fluid at the critical volume.

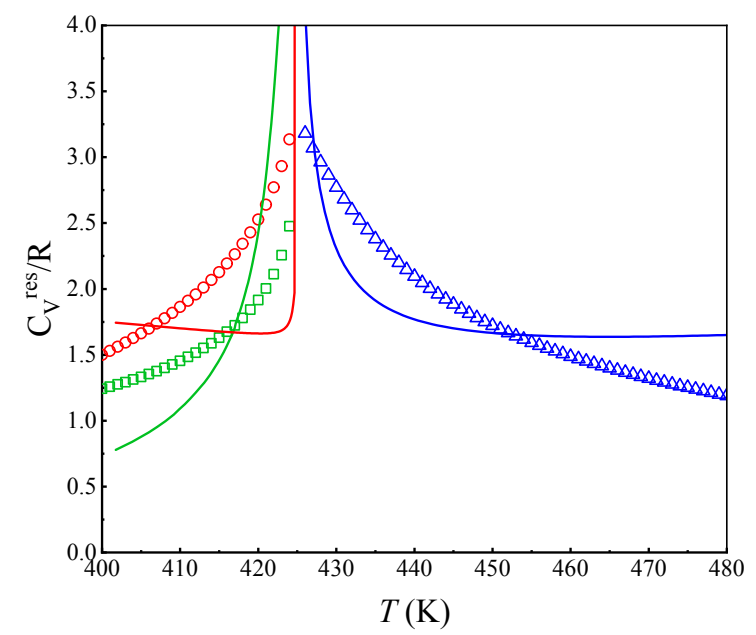

Fig. 11. The correlated experimental $C_{v}{ }^{\text {res }} / R$ for n-butane taken from [23], as well as the calculated curves using $S R K$ with Kiselev's method. The red and green lines and points represent the saturated vapor and liquid phases, respectively, while the blue lines and points describe the supercritical fluid at the critical volume. 
As previously done for $C_{P}$, we have applied the models to describe $C_{v}$ for different pressures close to the critical point. A summary of the main results of $C_{v}$ obtained with the crossover $E o S$ and the classical $S R K E O S$ is provided in Table 4 where $A A D \%$ is presented for selected constant pressures. Fig. 12 shows the results for the mean-field model and the non-mean-field model using Kiselev's method. It shows that the crossover equation corrects the behavior of the classical SRK EoS not only for the critical point, but also improves the description of the property below and above this point. This is due to the Kernel term, which provides the correct representation of the weak divergence. It is important to note that the classical model has an impact on the crossover SRK based on Kiselev's method. The figure indicates a descending trend below the critical temperature, which is intensified by the increase of pressure and carbon number of the hydrocarbon. This unphysical behavior is a limitation of the crossover SRK EOS based on Kiselev's approach to describe $C_{v}{ }^{r e s} / R$ at constant pressure in the asymptotic critical temperature range.

The crossover SRK EOS using White's method was also applied in the previous calculations. As expected, the approach similar to that of Cai and Prausnitz [26] is incapable of describing the weak divergence and the results are similar to the classical model, while the modified procedure is capable of representing the divergence (Fig. 13). Nevertheless, the comparison of this method with Kiselev's indicates that the Kernel term yields a superior description of this property, as it allows a correct description of the trend of the experimental data below the critical point. This behavior was also observed in other works using the crossover SAFT$V R$ [28] and Soft-SAFT [8] with the recursive procedure to include density fluctuations in the classical $E o S$. In both cases, the agreement for $C_{v}$ was not as accurate as the other properties, since the Kernel term was not applied to represent the weakly divergent behavior of the property. 


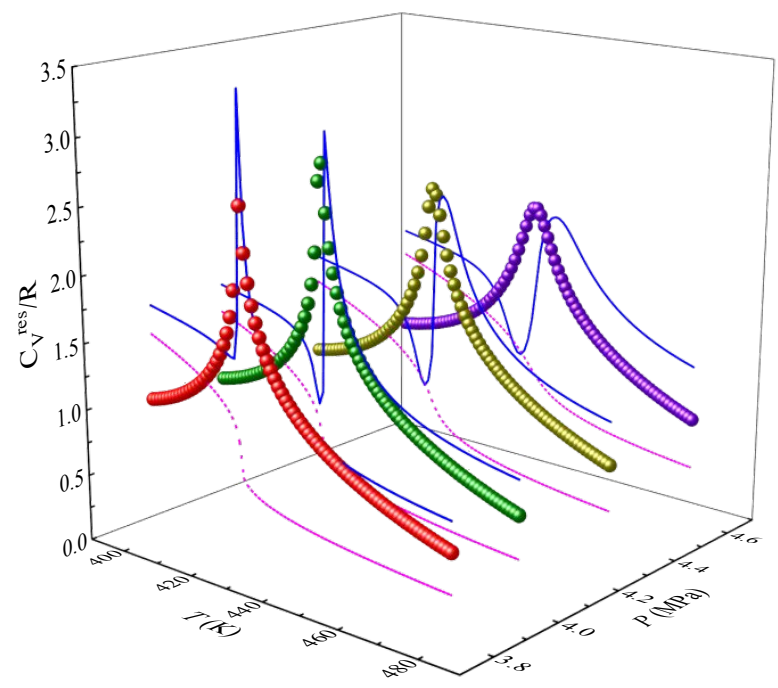

Fig. 12. Experimental $C_{v}{ }^{r e s} / R$ for $n$-butane at the pressures shown in Table 4 taken from [23]. The dashed lines correspond to the classical SRKEOS and the solid lines to the crossover model with Kiselev's method.

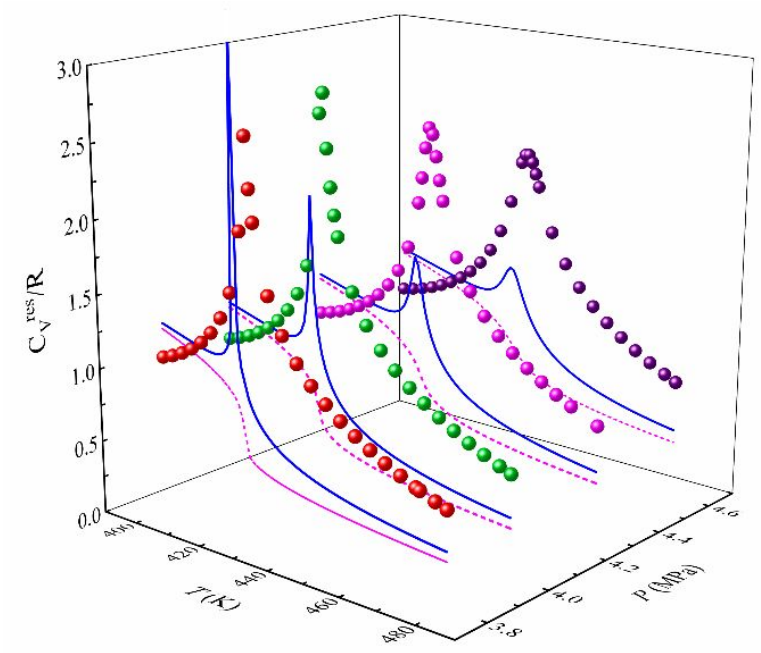

Fig. 13. Experimental $C_{v}{ }^{r e s} / R$ for n-butane at the pressures shown in Table 4 taken from [23]. The dashed and solid lines correspond to CSRK and CSRK2, respectively. 
A final test of the models for the representation of the heat capacity at constant volume was made by comparing the results of $C_{v}{ }^{r e s} / R$ along the critical density at different reduced temperatures for n-butane (Fig. 14). The plot is given in terms of the reduced temperatures to facilitate the comparison of the models, as the simulated critical temperature using White's method deviates slightly from the experimental one, and to evaluate whether the crossover EoSs are capable of capturing the divergence at the critical temperature [32]. Fig.14 clearly shows that the second approach of recursive procedure (CSRK2) possesses a non-mean-field behavior. Moreover, the $A A D \%$ between the simulated $C_{v}$ and the correlated supercritical data [23] is almost half of the first method, i.e. $5.26 \%$ and $10.15 \%$, respectively. In addition, Fig. 14 also depicts the capability of the crossover $S R K E O S$ based on Kiselev's approach to reproduce the divergence of $C_{v}{ }^{r e s} / R$ at the critical temperature. The calculated values of $C_{v}{ }^{r e s} / R$ along the critical density for n-butane show satisfactory agreement with the correlated experimental data and the $A A D \%$ for this isochore is $1.60 \%$. The comparison of the crossover EoS using Kiselev's approach and the second approach of the recursive procedure indicates that the Kernel term is important to describe the asymptotic behavior of $C_{v}{ }^{r e s} / R$ near the critical region.

Finally, we applied the models to represent the speed of sound $\left(V_{s}\right)$ of the selected hydrocarbons studied in this work. This property is related to both types of heat capacity and it is correct representation is important in several processes [25]. Since the classical model is not capable of describing the weak divergence related to the heat capacity at constant volume, it is expected some inferior results for the representation of the speed of sound in comparison to the crossover procedures. 


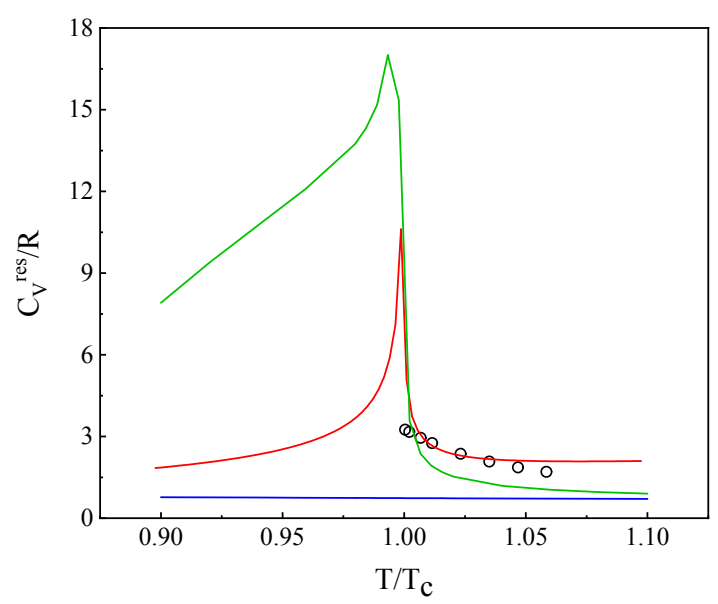

Fig. 14. Experimental $C_{v}{ }^{r e s} / R$ for n-butane at different reduced temperatures along the critical density close to the critical point. The blue, green and red lines correspond to CSRK, CSRK2 and Kiselev's approach, respectively. The correlated experimental data was taken from [23].

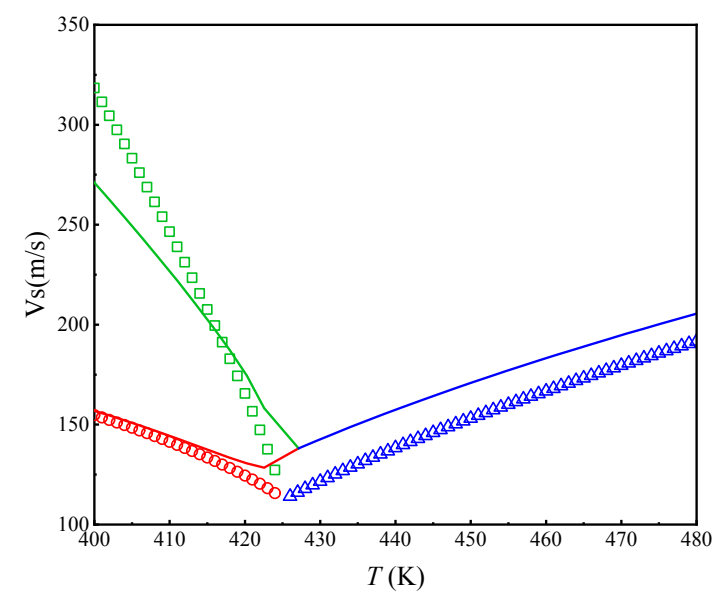

Fig. 15. The correlated experimental $V_{s}$ for n-butane taken from [23], as well as the calculated curves using the SRK EOS. The red and green lines and points represent the saturated vapor and liquid phases, respectively, while the blue lines and points describe the supercritical fluid at the critical volume. 
Fig. 15 shows the experimental data for the speed of sound of n-butane and the results obtained with the classical SRK EoS. The comparison of the predicted curves with the experimental data shows that the larger deviations occur at the critical point, which is related to the limitation of the model to represent the weak divergence of the specific heat capacity at constant volume. The same behavior is observed when the first approach of the recursive procedure is applied to incorporate the density fluctuations into the classical $E o S$ (Fig. 16). On the other hand, the second approach improves the results, yielding better agreement near the critical point. The same is observed when Kiselev's method is applied (Fig.17).

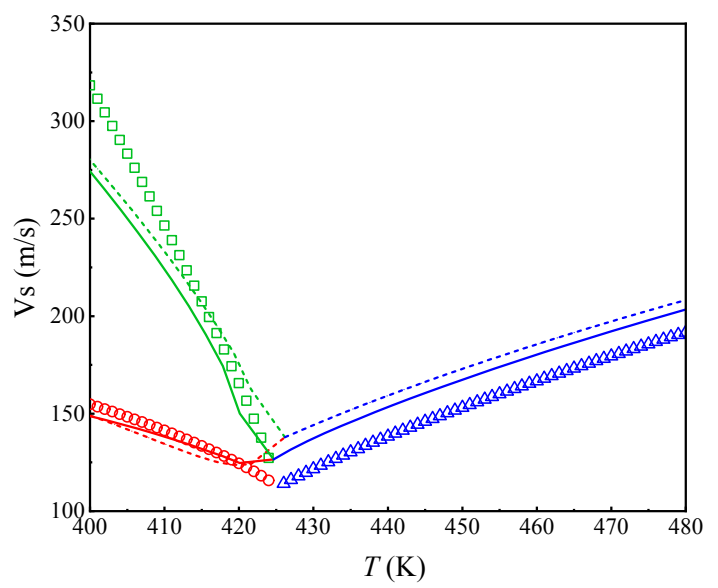

Fig. 16. The correlated experimental $V_{S}$ for n-butane taken from [23], as well as the calculated curves using the crossover $S R K E O S$ with both approached of the recursive procedure. The dashed and solid lines correspond to CSRK and CSRK2, respectively. The red and green lines and points represent the saturated vapor and liquid phases, respectively, while the blue lines and points describe the supercritical fluid at the critical volume.

The comparison of the crossover EoS using Kiselev's method and the second approach of the recursive procedure indicates that the Kernel term is important for correcting the classical model 
in the critical region. Fig. 17 shows a positive deviation of $C_{v}^{r e s} / R$, while there is a negative deviation when the other equations are applied.

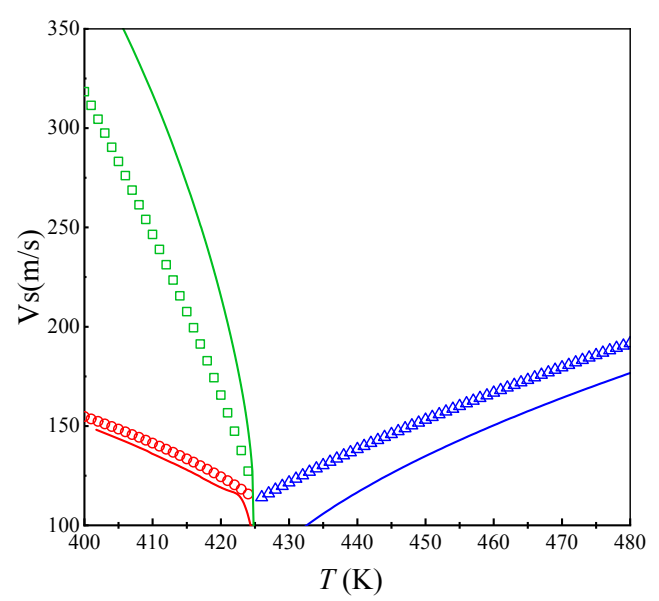

Fig. 17. The correlated experimental $V_{s}$ for n-butane taken from [23], as well as the calculated curves using the crossover $S R K E O S$ with Kiselev's method. The red and green lines and points represent the saturated vapor and liquid phases, respectively, while the blue lines and points describe the supercritical fluid at the critical volume.

Following the previous calculations, we applied the models to describe $V_{s}$ of the properties for different pressures close to the critical point. Fig. 18 shows the comparison between the classical SRK and the corrected model using Kiselev's method. Although the results are similar for supercritical conditions, superior results are obtained with the crossover model at the critical point and for the saturated liquid phase. On the other hand, the comparison of the mean-field model with the crossover SRK using White's method (Fig. 19) indicates that the second approach yields similar results at the critical point, but is inferior to the other $R G$ method for the description of the property in subcritical conditions. According to the summary of the main results of $V_{S}$ obtained with the crossover EoSs and the classical SRK EOS reported in Table 5, the importance of the Kernel term 
to incorporate the short wavelength fluctuations not only at the divergence but also close to the critical point can be reflected [25].

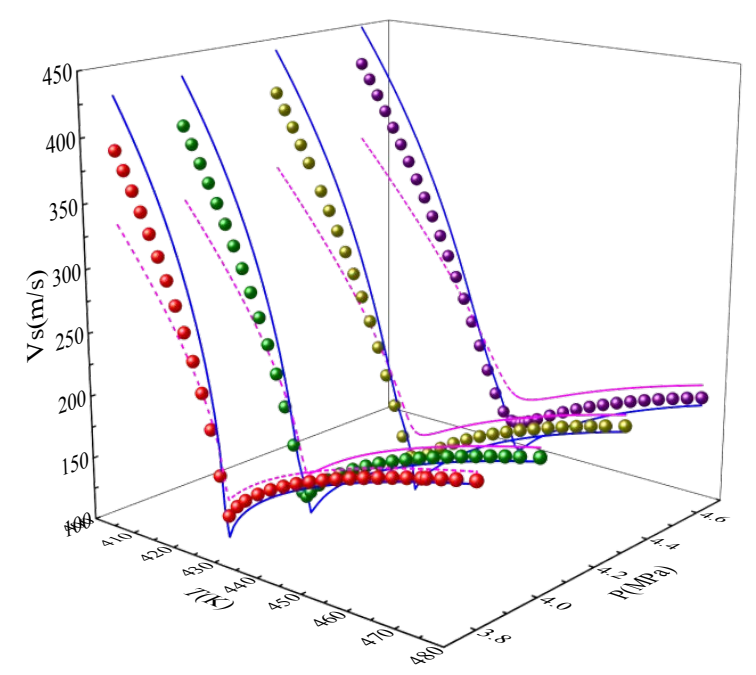

Fig. 18. Experimental $V_{s}$ at different pressures shown in Table 4 close to the critical point of nbutane, as well as the calculated curves using the classical SRK EOS (dash lines) and the model corrected with Kiselev's method (solid lines). The correlated experimental data was taken from [23].

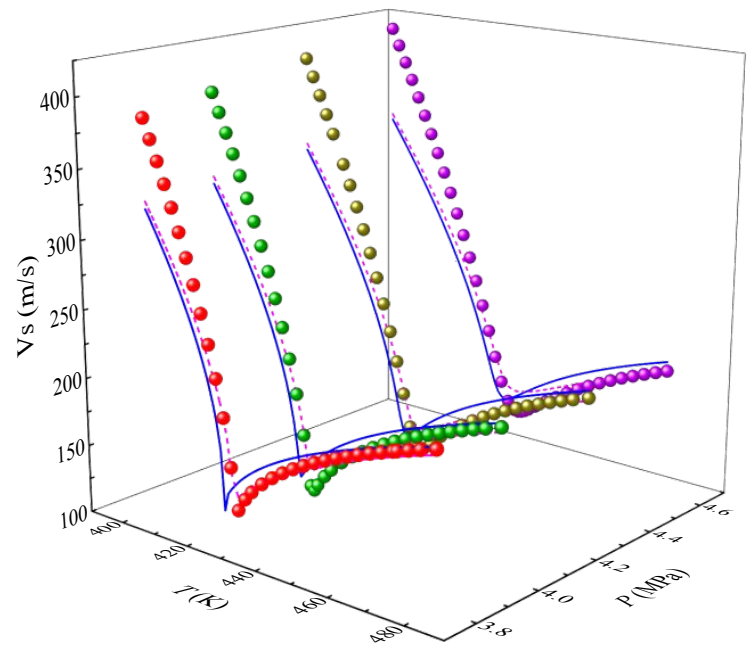

Fig. 19. Experimental $V_{s}$ at different pressures shown in Table 4 close to the critical point of nbutane, as well as the calculated curves using the crossover $S R K E O S$ with two approaches of 
White's method. The dashed and solid lines correspond to CSRK and CSRK2, respectively. The correlated experimental data was taken from [23].

Table 5. Calculated deviations for $V s$ at constant pressures using different crossover methods applied on SRK EOS in compare to classical SRK EOS.

\begin{tabular}{|c|c|c|c|c|c|c|}
\hline Compound & $\mathrm{P}(\mathrm{MPa})$ & $\begin{array}{l}\mathrm{AAD} \% \\
\text { SRK }\end{array}$ & $\begin{array}{l}\text { AAD\% } \\
\text { Kiselev's } \\
\text { method }\end{array}$ & $\begin{array}{l}\text { AAD\% } \\
\text { White's } \\
\text { method } \\
\text { CSRK }\end{array}$ & $\begin{array}{l}\text { AAD\% } \\
\text { White's } \\
\text { method } \\
\text { CSRK2 }\end{array}$ & $\begin{array}{l}\text { T-range } \\
(\mathrm{K})\end{array}$ \\
\hline \multirow{4}{*}{$\mathrm{C} 1$} & 4.5992 & 5.7 & 4.9 & 4.0 & 3.8 & \multirow{4}{*}{$170-240$} \\
\hline & 4.8 & 6.6 & 5.6 & 5.1 & 4.6 & \\
\hline & 5.1 & 7.2 & 6.0 & 5.4 & 4.8 & \\
\hline & 5.4 & 7.6 & 6.5 & 5.7 & 5.2 & \\
\hline \multirow{4}{*}{$\mathrm{C} 2$} & 4.8718 & 6.7 & 2.6 & 4.9 & 4.8 & \multirow{4}{*}{$280-350$} \\
\hline & 5.0 & 7.1 & 3.3 & 5.3 & 5.2 & \\
\hline & 5.3 & 8.4 & 4.3 & 5.9 & 5.9 & \\
\hline & 5.6 & 9.1 & 4.5 & 6.4 & 6.5 & \\
\hline \multirow{4}{*}{$\mathrm{C} 3$} & 4.2476 & 4.7 & 3.9 & 3.4 & 4.2 & \multirow{4}{*}{$350-440$} \\
\hline & 4.5 & 5.9 & 2.7 & 3.5 & 5.1 & \\
\hline & 4.8 & 6.8 & 2.8 & 4.1 & 5.8 & \\
\hline & 5.1 & 7.6 & 2.9 & 4.5 & 6.2 & \\
\hline \multirow{4}{*}{$\mathrm{C} 4$} & 3.7960 & 5.9 & 6.8 & 4.0 & 7.7 & \multirow{4}{*}{$400-480$} \\
\hline & 4.0 & 7.1 & 7.0 & 4.4 & 8.6 & \\
\hline & 4.3 & 8.4 & 7.6 & 5.2 & 9.3 & \\
\hline & 4.6 & 7.2 & 2.7 & 6.2 & 9.5 & \\
\hline \multirow{4}{*}{$\mathrm{C} 5$} & 3.37 & 5.5 & 8.2 & 3.5 & 8.3 & \multirow{4}{*}{$450-520$} \\
\hline & 4.0 & 9.3 & 9.4 & 5.6 & 11.1 & \\
\hline & 4.3 & 10.5 & 9.1 & 7.2 & 11.4 & \\
\hline & 4.6 & 11.3 & 8.2 & 8.4 & 11.4 & \\
\hline \multirow{4}{*}{ C6 } & 3.034 & 7.9 & 11.7 & 5.8 & 14.4 & \multirow{4}{*}{$480-540$} \\
\hline & 3.3 & 10.1 & 11.8 & 7.5 & 16.5 & \\
\hline & 3.6 & 11.8 & 11.4 & 9.6 & 16.6 & \\
\hline & 3.9 & 12.7 & 9.6 & 10.9 & 15.6 & \\
\hline \multirow{4}{*}{ C7 } & 2.7360 & 7.0 & 10.8 & 1.7 & 12.2 & \multirow{4}{*}{$530-580$} \\
\hline & 3.0 & 9.2 & 11.4 & 3.4 & 15.6 & \\
\hline & 3.3 & 11.0 & 11.4 & 5.9 & 15.8 & \\
\hline & 3.6 & 11.9 & 9.8 & 7.7 & 14.6 & \\
\hline \multirow{4}{*}{$\mathrm{C} 8$} & 2.497 & 7.5 & 10.7 & 2.8 & 15.8 & \multirow{4}{*}{$550-600$} \\
\hline & 2.7 & 9.6 & 11.2 & 4.1 & 18.9 & \\
\hline & 3.0 & 11.4 & 11.5 & 6.2 & 18.6 & \\
\hline & 3.3 & 11.4 & 10.1 & 7.2 & 15.9 & \\
\hline
\end{tabular}




\begin{tabular}{|lllllll|}
\hline & 2.103 & 7.5 & 10.7 & 3.8 & 16.4 & \\
C10 & 2.4 & 9.6 & 11.2 & 6.6 & 20.5 & $590-650$ \\
& 2.7 & 11.4 & 11.5 & 20.3 & 19.4 & \\
\hline Average & 3.0 & 11.4 & 10.1 & 17.2 & 15.9 & \\
\hline
\end{tabular}

The calculation of the critical exponent is an important test to evaluate the behavior of the $E o S$, as the mean-field models are unable to predict the correct values of the universal critical exponents. In this work, we focus on the estimation of the exponent $\alpha$ using the following expression:

$$
\ln \left(C_{v}\right)=-\alpha \ln (\Delta T)+b
$$

where $\alpha$ is the slope of the linear regression of the natural logarithm of $C_{v}$ as a function of $\Delta T$, which is $\left|T-T_{c}\right| / T_{c}$. Along the critical isochore, $C_{v}$ asymptotically behaves proportionally to $\Delta$ $T^{-\alpha}$, and the universal value of $\alpha$ is equal to approximately 0.11 [25]. On the other hand, the meanfield approximation predicts a value equal to 0 for this exponent.

Fig. 20 shows the critical exponent $\alpha$ calculated in the temperature range between $0.98 T_{c}$ and $T_{C}$ with both crossover methods. It is possible to observe that CSRK1 yields the classical value, while $\alpha$ simulated with CSRK2 is close to the universal value. As explained previously, the critical behavior of the heat capacity at constant volume is determined by short-range interactions, hence the addition of the long-range term in CSRK1 limits the incorporation of longer wavelength density fluctuations resulting in a critical exponent similar to the classical one. It is important to note that increasing the summand order does not modify this behavior. Besides, Kiselev's model cannot represent the divergent behavior of the isochoric heat capacity only based on the rescaling of the physical variables. In fact, it contains the Kernel term, an additive term related to the divergent behavior of $C_{v}$ at the critical isochore, which normally improves the results for exponent $\alpha$ [9]. 


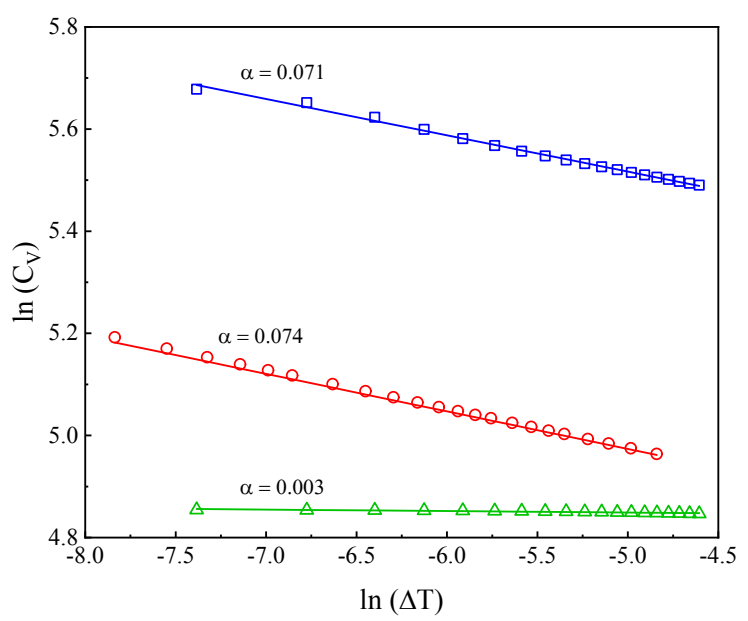

Fig. 20. Natural logarithm of the heat capacity at constant volume as a function of the dimensionless temperature distance for n-butane determined with the CSRK1 (green triangles), CSRK2 (blue squares) and Kiselev's (red circles) models. Critical exponent alpha is calculated from the slope of the linear regression of each model (solid lines).

In order to show the effect of the number of iterations in the representation of the derivative properties, a plot of $C_{p}^{r e s} / R$ and $C_{v}^{r e s} / R$ at the critical pressure is given in Fig. 21 . The difference between the curves is marginal, as the corrections of the energy density after 5 iterations are marginal. Nonetheless, a small improvement is seen for $C_{p}^{r e s} / R$ in comparison to $C_{v}^{r e s} / R$. This behavior is related to the fact that $C_{v}$ is determined by the short -range interactions which are limited in CSRK1. 


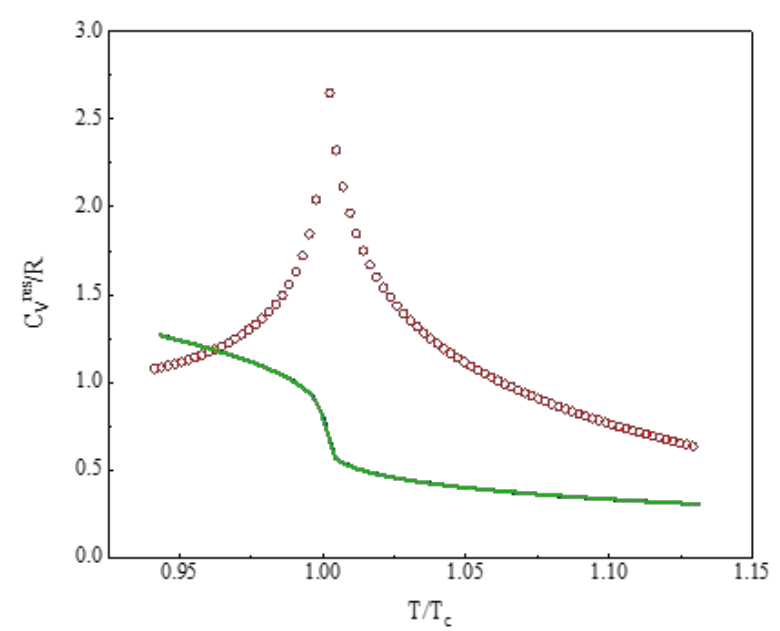

(a)

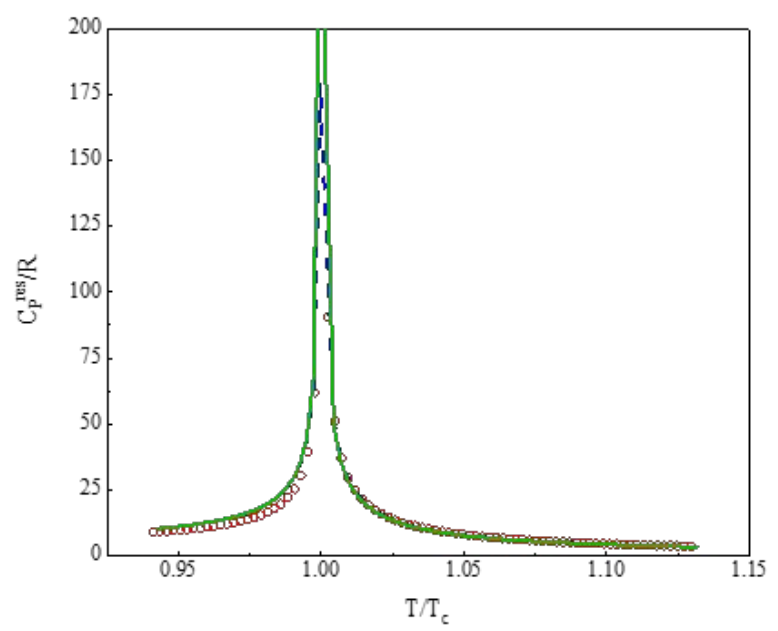

(c)

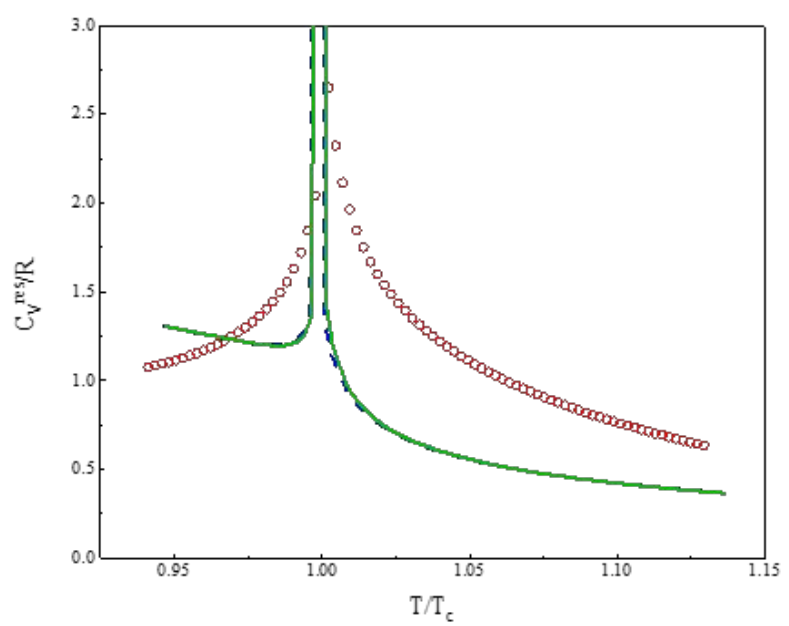

(b)

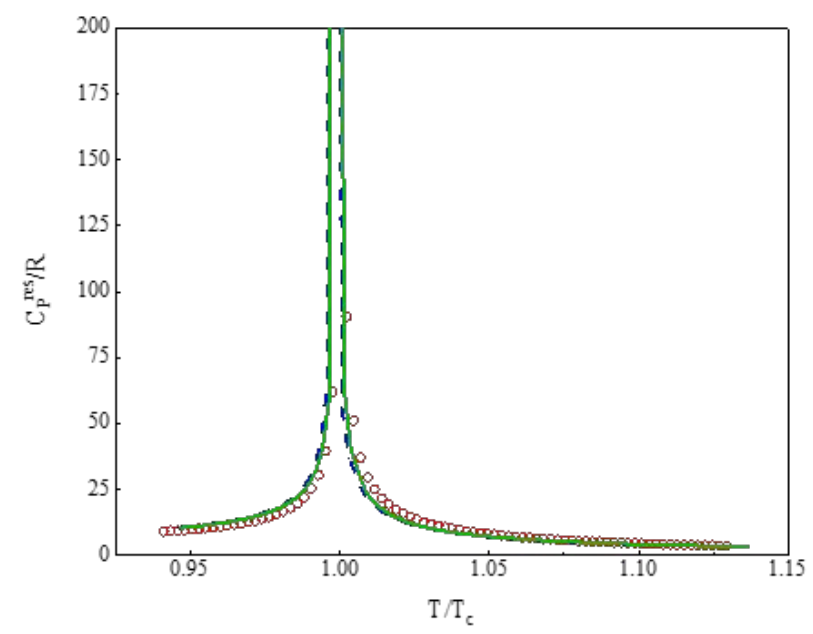

(d)

Fig. 21. The correlated experimental $C_{v}{ }^{r e s} / R(\mathrm{a}$ and $\mathrm{b})$ and $C_{p}{ }^{r e s} / R(\mathrm{c}$ and $\mathrm{d})$ for n-butane at critical pressure taken from [23]. Figures (a) and (c) show the calculated curves using CSRK1, while figures (b) and (d) are the results obtained with CSRK2, using 5 (solid lines) and 7 (dashed lines) iterations for the calculation of the free energy density function in White's procedure. 


\section{CONCLUSIONS}

In this work, we applied two different methods to correct the classical SRK EoS for improving the representation of derivative properties in the critical region. First we used a procedure based on the Landau-Ginzburg expansion developed by Kiselev and co-workers [13-16,32,37]. Then we have used two different approaches based on White's recursive procedure. In both cases it was possible to observe an improvement of the results due to the incorporation of density fluctuations into the mean-field equation. Although the improvements are not substantial for the overall $A A D \%$ in comparison to the classical model, a superior description of the qualitative behavior of the derivative properties, specially for the isochoric heat capacity, is observed.

We have also shown that the procedure developed by Cai and Prausnitz [26] limits the introduction of short wavelengths, which hinders the description of the properties related to the weak divergence at the critical point, like the molar heat capacity at constant volume and the speed of sound. This behavior can be corrected by modifying the procedure in a way similar to that is used by many implementations in SAFT-type $\operatorname{EoS}[28,29]$.

The models were then used to describe the heat capacity at constant pressure and volume, as well as the speed of sound for selected n-alkanes. The comparison of the equations with experimental data showed that the crossover models yield similar results to the mean-field one for $C_{p}^{r e s}$ due to the strong divergence of this property. On the other hand, in the case of $C_{v}^{r e s}$, only the non-mean-field models based on Kiselev's and the second approach of White's recursive procedure were capable of predicting the weak divergence related to this property. This is the reason the non-mean-field models are also superior in the description of the speed of sound.

Finally, the comparison of White's and Kiselev's method indicates that the Kernel term is important for describing the $C_{v}^{r e s}$ and the speed of sound close to and far away from the critical 
region, since, although the crossover $S R K E O S$ using White's procedure is capable of capturing the divergence of the property at the critical point, the mean-field model using Kiselev's method has a superior match regarding the trend of data in sub-critical conditions.

\section{ACKNOWLEDGMENT}

The authors gratefully acknowledge the financial support from Center for Energy Resources Engineering (CERE) at Technical University of Denmark (DTU), the Science Without Borders Program of the Brazilian Ministry of Education for paying the scholarship of Andre P.C.M. Vinhal and the Research Committee of University of Tehran. 


\section{REFERENCES}

[1] J.. Rowlinson, Molecular thermodynamics of fluid-phase equilibria, J. Chem. Thermodyn. 2 (2004) 158-159. doi:10.1016/0021-9614(70)90078-9.

[2] E. Hendriks, G.M. Kontogeorgis, R. Dohrn, J.-C. de Hemptinne, I.G. Economou, L.F. Žilnik, V. Vesovic, Industrial Requirements for Thermodynamics and Transport Properties, Ind. Eng. Chem. Res. 49 (2010) 11131-11141. doi:10.1021/ie101231b.

[3] M. Lagache, P. Ungerer, A. Boutin, A.H. Fuchs, Prediction of thermodynamic derivative properties of fluids by Monte Carlo simulation, Phys. Chem. Chem. Phys. 3 (2001) 43334339. doi:10.1039/b104150a.

[4] J.C. de Hemptinne, B.A. P. Ungerer, E. Behar, Thermodynamic Modeling and Materials Data Engineering, in: J. Caliste, A. Truyol, J. Westbrook (Eds.), Thermodyn. Model. Mater. Data Eng., Springer-Verlag, Berlin, 1998: pp. 53-70.

[5] J. V Sengers, J.M.H.L. Sengers, Thermodynamic Behavior of Fluids Near the Critical Point, Annu. Rev. Phys. Chem. 37 (1986) 189-222. doi:10.1146/annurev.pc.37.100186.001201.

[6] K.G. Wilson, Renormalization Group and Critical Phenomena. I. Renormalization Group and the Kadanoff Scaling Picture, Phys. Rev. B. 4 (1971) 3174-3183. doi:10.1103/PhysRevB.4.3174.

[7] A.K. Wyczalkowska, J. V Sengers, M.A. Anisimov, Critical fluctuations and the equation of state of Van der Waals, Phys. A Stat. Mech. Its Appl. 334 (2004) 482-512. doi:https://doi.org/10.1016/j.physa.2003.11.021.

[8] F. Llovell, L.F. Vega, Global fluid phase equilibria and critical phenomena of selected 
mixtures using the crossover soft-SAFT equation, J. Phys. Chem. B. 110 (2006) 1350-1362. doi:10.1021/JP0551465.

[9] P.C. Albright, Z.Y. Chen, J. V Sengers, Crossover from singular to regular thermodynamic behavior of fluids in the critical region, Phys. Rev. B. 36 (1987) 877-880. doi:10.1103/PhysRevB.36.877.

[10] J. Janeček, P. Paricaud, M. Dicko, C. Coquelet, A generalized Kiselev crossover approach applied to Soave-Redlich-Kwong equation of state, Fluid Phase Equilib. 401 (2015) 16-26. doi:10.1016/j.fluid.2015.04.024.

[11] A. Shen, Q. Liu, Y. Duan, Z. Yang, Crossover equation of state for selected hydrocarbons (C4-C7), Chinese J. Chem. Eng. 22 (2014) 1291-1297. doi:10.1016/j.cjche.2014.09.013.

[12] X.-H. Xu, Y.-Y. Duan, Z. Yang, Crossover Volume Translation Soave-Redlich-Kwong Equation of State for Fluids, Ind. Eng. Chem. Res. 51 (2012) 6580-6585. doi:10.1021/ie300112j.

[13] S.B. Kiselev, Cubic crossover equation of state1, Fluid Phase Equilib. 147 (1998) 7-23.

[14] S.B. Kiselev, D.G. Friend, Cubic crossover equation of state for mixtures1Contribution of the National Institute of Standards and Technology, not subject to copyright in the United States.1, Fluid Phase Equilib. 162 (1999) 51-82. doi:https://doi.org/10.1016/S03783812(99)00182-X.

[15] S.B. Kiselev, J.F. Ely, Simplified crossover SAFT equation of state for pure fluids and fluid mixtures, Fluid Phase Equilib. 174 (2000) 93-113. doi:10.1016/S0378-3812(00)00420-9.

[16] S.B. Kiselev, J.F. Ely, S.P. Tan, H. Adidharma, M. Radosz, HRX-SAFT equation of state 
for fluid mixtures: Application to binary Mixtures of carbon dioxide, water, and methanol, Ind. Eng. Chem. Res. 45 (2006) 3981-3990. doi:10.1021/ie0512082.

[17] K.G. Wilson, M.E. Fisher, Critical Exponents in 3.99 Dimensions, Phys. Rev. Lett. 28 (1972) 240-243. doi:10.1103/PhysRevLett.28.240.

[18] J.A. White, S. Zhang, Renormalization group theory for fluids, J. Chem. Phys. 99 (1993) 2012-2019. doi:10.1063/1.465263.

[19] J.A. White, S. Zhang, Renormalization theory of nonuniversal thermal properties of fluids, J. Chem. Phys. 103 (1995) 1922-1928. doi:10.1063/1.469716.

[20] J.A. White, Contribution of fluctuations to thermal properties of fluids with attractive forces of limited range: theory compared with $\mathrm{PQT}$ and $\mathrm{Cv}$ data for argon, Fluid Phase Equilib. 75 (1992) 53-64. doi:https://doi.org/10.1016/0378-3812(92)87006-9.

[21] L.W. Salvino, J.A. White, Calculation of density fluctuation contributions to thermodynamic properties of simple fluids, J. Chem. Phys. 96 (1992) 4559-4568.

[22] J.A. White, Global renormalization calculations compared with simulations for LennardJones fluid, J. Chem. Phys. 112 (2000) 3236-3244. doi:10.1063/1.480907.

[23] M. Lemmon, E. , Huber, M., McLinden, NIST, Standard Reference Data Program, Gaithersburg. (2013).

[24] A. Anderko, 4 Cubic and generalized van der waals equations, in: J. V Sengers, R.F. Kayser, C.J. Peters, H.J.B.T.-E.T. White (Eds.), Equations State Fluids Fluid Mix., Elsevier, 2000: pp. 75-126. doi:https://doi.org/10.1016/S1874-5644(00)80015-6. 
[25] F. Llovell, L.F. Vega, M.A. Anisimov, J. V. Sengers, Incorporating critical divergence of isochoric heat capacity into the soft-SAFT equation of state, AIChE J. 61 (2015) 30733080. doi:10.1002/aic.14911.

[26] J. Cai, J.M. Prausnitz, Thermodynamics for fluid mixtures near to and far from the vaporliquid critical point, Fluid Phase Equilib. 219 (2004) 205-217. doi:10.1016/j.fluid.2004.01.033.

[27] A. P. C. M. Vinhal, W. Yan, G.M. Kontogeorgis, Application of a Crossover Equation of State to Describe Phase Equilibrium and Critical Properties of $\mathrm{n}$-Alkanes and Methane/ $\mathrm{n}$ Alkane Mixtures, J. Chem. Eng. Data. 63 (2018) 981-993. doi:10.1021/acs.jced.7b00779.

[28] E. Forte, F. Llovell, L.F. Vega, J.P.M. Trusler, A. Galindo, Application of a renormalization-group treatment to the statistical associating fluid theory for potentials of variable range (SAFT-VR), J. Chem. Phys. 134 (2011) 2012. doi:10.1063/1.3570614.

[29] E. Forte, F. Llovell, J.P.P.M. Trusler, A. Galindo, Application of the statistical associating fluid theory for potentials of variable range (SAFT-VR) coupled with renormalisationgroup $(\mathrm{RG})$ theory to model the phase equilibria and second-derivative properties of pure fluids, Fluid Phase Equilib. 337 (2013) 274-287. doi:10.1016/j.fluid.2012.09.022.

[30] A. Jamali, A.P.C.M. Vinhal, H. Behnejada, W. Yan, G.M. Kontogeorgis, Comparison of two crossover procedures for describing thermodynamic behavior of normal alkanes from singular critical to regular classical regions. Manuscript submitted for publication, Fluid Phase Equilib. 495 (2019) 33-46. https://doi.org/10.1016/j.fluid.2019.04.030.

[31] M.S. Shin, Y. Lee, H. Kim, Estimation of second-order derivative thermodynamic 
properties using the crossover cubic equation of state, J. Chem. Thermodyn. 40 (2008) 688694. doi:10.1016/J.JCT.2007.10.008.

[32] S.B. Kiselev, J.F. Ely, I.M. Abdulagatov, J.W. Magee, Crossover SAFT equation of state and thermodynamic properties of propan-1-ol, Int. J. Thermophys. 21 (2000) 1373-1405.

[33] O. Vilaseca, F. Llovell, J. Yustos, R.M. Marcos, L.F. Vega, Phase equilibria, surface tensions and heat capacities of hydrofluorocarbons and their mixtures including the critical region, J. Supercrit. Fluids. 55 (2010) 755-768. doi:10.1016/J.SUPFLU.2010.10.015.

[34] A.M.A. Dias, F. Llovell, J.A.P. Coutinho, I.M. Marrucho, L.F. Vega, Thermodynamic characterization of pure perfluoroalkanes, including interfacial and second order derivative properties, using the crossover soft-SAFT EoS, Fluid Phase Equilib. 286 (2009) 134-143. doi:10.1016/J.FLUID.2009.08.018.

[35] F. Llovell, L.F. Vega, Phase equilibria, critical behavior and derivative properties of selected n-alkane/n-alkane and n-alkane/1-alkanol mixtures by the crossover soft-SAFT equation of state, J. Supercrit. Fluids. $41 \quad$ (2007) 204-216. doi:10.1016/J.SUPFLU.2006.10.001.

[36] F. Llovell, C.J. Peters, L.F. Vega, Second-order thermodynamic derivative properties of selected mixtures by the soft-SAFT equation of state, Fluid Phase Equilib. 248 (2006) 115122.

[37] S.B. Kiselev, I.G. Kostyukova, A.A. Povodyrev, Universal crossover behavior of fluids and fluid mixtures in the critical region, Int. J. Thermophys. 12 (1991) 877-895. 


\section{TABLE OF CONTENTS (TOC)/ABSTRACT GRAPHIC}

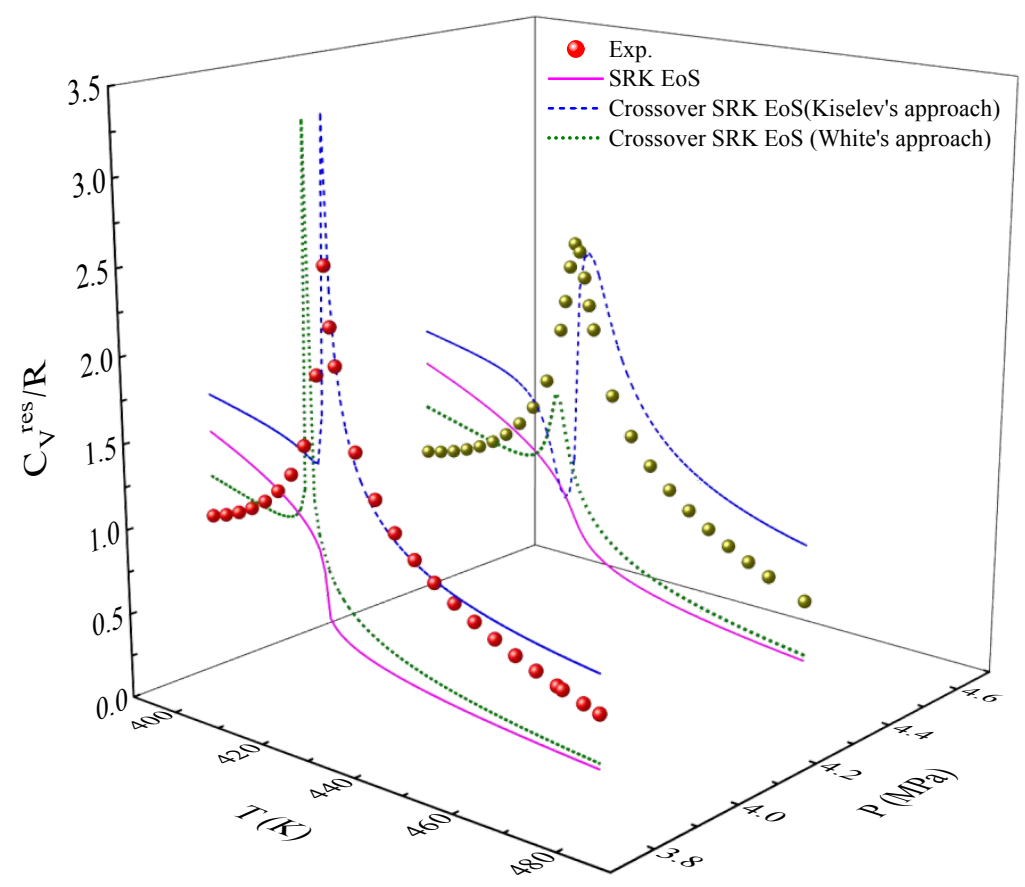

ACS Paragon Plus Environment 